\title{
Adaptor proteins NUMB and NUMBL promote cell cycle withdrawal by targeting ERBB2 for degradation
}

\author{
Maretoshi Hirai, ${ }^{1}$ Yoh Arita, ${ }^{1}$ C. Jane McGlade, ${ }^{2}$ Kuo-Fen Lee, ${ }^{3}$ Ju Chen, ${ }^{4}$ and Sylvia M. Evans ${ }^{1,4,5}$ \\ 'Skaggs School of Pharmacy and Pharmaceutical Sciences, UCSD, La Jolla, California, USA. ²Program in Cell Biology, The Hospital for Sick Children, Toronto, Ontario, Canada. \\ ${ }^{3}$ Clayton Foundation Laboratories for Peptide Biology, The Salk Institute, La Jolla, California, USA. ${ }^{4}$ Department of Medicine and ${ }^{5}$ Department of Pharmacology, UCSD, La Jolla, California, USA.
}

\begin{abstract}
Failure of trabecular myocytes to undergo appropriate cell cycle withdrawal leads to ventricular noncompaction and heart failure. Signaling of growth factor receptor ERBB2 is critical for myocyte proliferation and trabeculation. However, the mechanisms underlying appropriate downregulation of trabecular ERBB2 signaling are little understood. Here, we have found that the endocytic adaptor proteins NUMB and NUMBL were required for downregulation of ERBB2 signaling in maturing trabeculae. Loss of NUMB and NUMBL resulted in a partial block of late endosome formation, resulting in sustained ERBB2 signaling and STAT5 activation. Unexpectedly, activated STAT5 overrode Hippo-mediated inhibition and drove YAP1 to the nucleus. Consequent aberrant cardiomyocyte proliferation resulted in ventricular noncompaction that was markedly rescued by heterozygous loss of function of either ERBB2 or YAP1. Further investigations revealed that NUMB and NUMBL interacted with small GTPase Rab7 to transition ERBB2 from early to late endosome for degradation. Our studies provide insight into mechanisms by which NUMB and NUMBL promote cardiomyocyte cell cycle withdrawal and highlight previously unsuspected connections between pathways that are important for cardiomyocyte cell cycle reentry, with relevance to ventricular noncompaction cardiomyopathy and regenerative medicine.
\end{abstract}

\section{Introduction}

The growth factor receptor ERBB2 plays a pivotal role in promoting cardiomyocyte proliferation and formation of trabecular myocytes during early heart development $(1,2)$. Activation of myocardial ERBB2/ERBB4 is mediated by neuregulin secreted from endocardium $(1,3)$. However, mechanisms by which ERBB2 signaling is downregulated as trabecular myocytes undergo cell cycle withdrawal have not been addressed. Failure of trabecular myocytes to undergo cell cycle withdrawal can result in noncompaction cardiomyopathy, a sometimes fatal heart disease (4).

The endocytic adaptor protein NUMB shares homology with a family of cargo-selective endocytic adaptors (5). NUMB inhibits Notch signaling, but mechanisms by which it does so are controversial, with some data suggesting that NUMB positively regulates endocytosis of NOTCH1 and other data suggesting that NUMB negatively regulates endocytic recycling of NOTCH1 $(6,7)$. On the other hand, recent in vivo imaging in Drosophila demonstrated that NUMB inhibits Notch by targeting sanpodo, a Notch partner, to late endosomes (LEs) (8). Other cargoes of NUMB include the transferrin receptor and integrins $(5,9)$. Detailed insight into mechanisms by which NUMB regulates trafficking of other cargoes is lacking (10).

Here, we explored mechanisms by which loss of NUMB or both NUMB and NUMB-like (NUMBL) in cardiomyocytes results in ventricular noncompaction phenotypes. Our results elucidate

Conflict of interest: The authors have declared that no conflict of interest exists Submitted: October 6, 2016; Accepted: November 10, 2016

Reference information: J Clin Invest. 2017;127(2):569-582.

https://doi.org/10.1172/JCI91081. a detailed pathway by which ERBB2 signaling is downregulated and reveal a STAT5/YAP pathway activated when ERBB2 signaling is aberrantly sustained that can override Hippo-mediated cardiomyocyte cell cycle withdrawal. Our work identifies pathways worthy of future investigation in the setting of noncompaction cardiomyopathy and pathways that have relevance for promoting cardiomyocyte cell cycle reentry in the context of regenerative medicine. Additionally, we discovered an interaction of NUMB with Rab7, giving new insight into mechanisms by which NUMB and NUMBL act at the early to late endosomal interface to promote growth factor degradation and cell cycle withdrawal. Our findings are also likely to have a bearing on the role of NUMB and NUMBL as tumor suppressors $(11,12)$.

\section{Results}

Myocardial loss of $\mathrm{Nb}$ alone or $\mathrm{Nb} / \mathrm{Nbl}$ results in ventricular noncompaction. To explore the role of NUMB (encoded by $\mathrm{N} b$ ) and NUMBL (encoded by $\mathrm{Nbl}$ ) in cardiomyocytes, we utilized troponin $\mathrm{T}-$ Cre (Tnnt-Cre, hereafter referred to as TnT-Cre) to ablate either $\mathrm{Nb}$ alone ( $\mathrm{Nb} \mathrm{cKO})$ or both $\mathrm{Nb}$ and $\mathrm{Nbl}$ ( $\mathrm{Nb} / \mathrm{NbL} \mathrm{cKO})(13-15)$. Lineage studies with the R26-mTmG membrane indicator (16) demonstrated efficient cardiomyocyte-specific ablation at E10.5 (Supplemental Figure 1A; supplemental material available online with this article; https://doi.org/10.1172/JCI91081DS1). Immunostaining for NUMB confirmed cardiomyocyte-specific loss of NUMB at E10.5 (Supplemental Figure 1B). Observed cardiac phenotypes with double cKOs were consistent with previous studies utilizing myocardial Cres to ablate both $\mathrm{Nb}$ and $\mathrm{Nbl}(17,18)$ (Figure 1, A and B). We also observed similar, but less severe, cardiac phenotypes in $\mathrm{Nb}$ cKO alone (Figure $1, \mathrm{~A}$ and $\mathrm{B}$ ). 
A

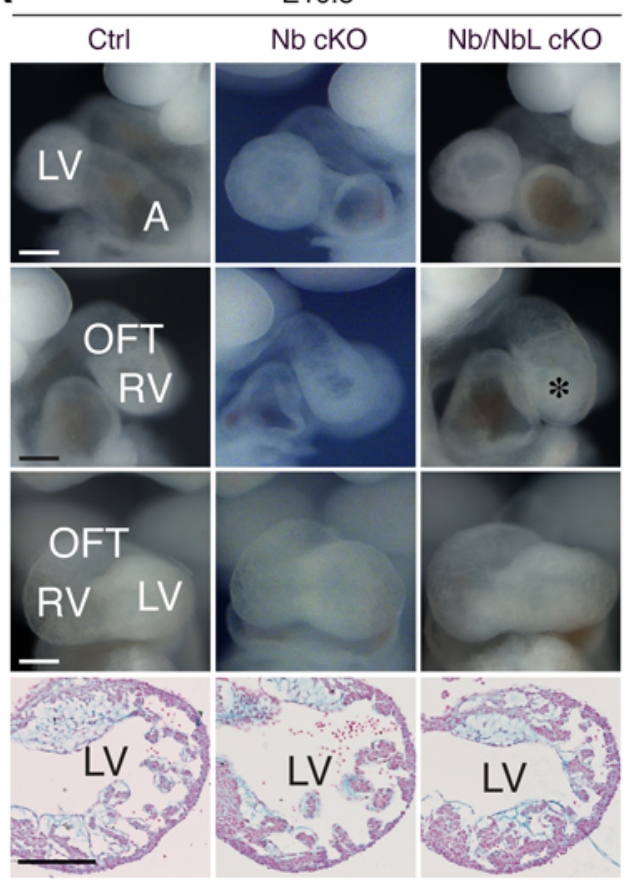

B

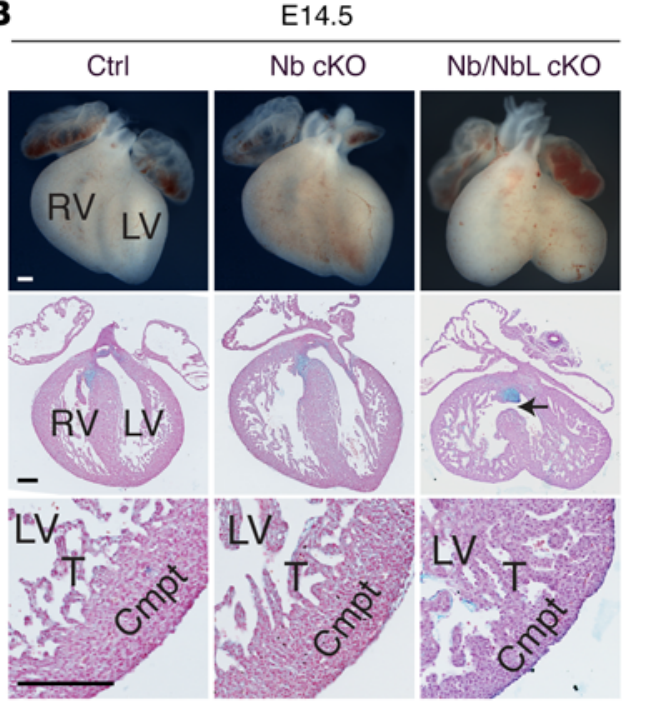

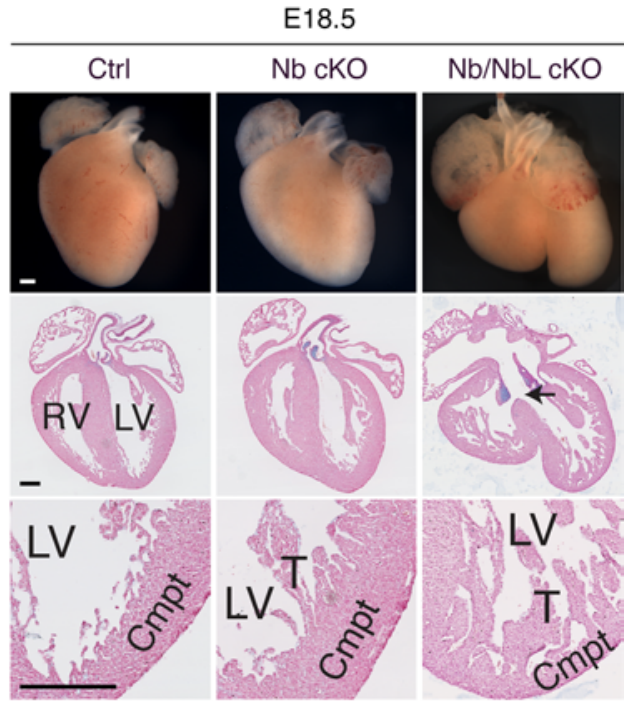

Figure 1. Abnormal trabeculae and increased proliferation of trabeculae in $\mathrm{Nb}$ or $\mathrm{Nb} / \mathrm{NbL}$ cKOs. (A and B) Wholemount views and sections stained with nuclear fast red and Alcian blue. Asterisk indicates dead cells in interventricular septum. Arrows indicate ventricular septal defect. Scale bars: $200 \mu \mathrm{m}$. Ctrl, control; OFT, outflow tract; T, trabeculae; Cmpt, compact layer.

At E10.5, by whole mount, $\mathrm{Nb}$ or $\mathrm{Nb} / \mathrm{NbL}$ cKO hearts did not appear much different from controls. However, sections revealed thicker trabeculae in $\mathrm{Nb}$ and $\mathrm{Nb} / \mathrm{NbL}$ cKOs (Figure 1A). At E14.5, $\mathrm{Nb} / \mathrm{NbL} \mathrm{cKO}$ hearts were shaped abnormally, with an abnormal groove at the interventricular septum (IVS) (Figure 1B). Sections revealed thicker trabeculae and thinner ventricular walls in $\mathrm{Nb}$ cKOs, with more extreme phenotypes in double cKOs (Figure 1B). Double cKOs exhibited ventricular septal defects (VSDs) at E14.5 and E18.5 (Figure 1B).

Immunostaining of E10.5 and E12.5 heart sections for tropo$\operatorname{nin} \mathrm{T}$ and quantitative analyses confirmed trabecular phenotypes

(Supplemental Figure 1, C-F) and revealed disorganized myofibrillar structure at E10.5 that was more organized by E12.5, although quantitative analyses revealed that sarcomeres within mutant trabeculae remained less developed than those of controls (Supplemental Figure 1, G-I). TUNEL staining to examine apoptosis did not reveal significant differences between $\mathrm{Nb}$ / $\mathrm{NbL} \mathrm{cKO}$ and control hearts, other than a small region of dead cells within the ventricular septum of $\mathrm{Nb} / \mathrm{NbL}$ cKOs (Supplemental Figure 1C).

The above phenotypes suggested a lack of normal trabecular cell cycle withdrawal in mutants. To examine cardiomyocyte proliferation, E10.5 sections from embryos that had been labeled by EdU were immunostained with antibodies to troponin $\mathrm{T}$ and CD31 and stained for EdU and DAPI. As expected, control trabecular nuclei exhibited EdU labeling in less than $5 \%$ of cardiomyocytes, consistent with reduced cell cycle activity by E10.5 (Figure 2A). In contrast, Nb cKO trabeculae exhibited a significant increase in EdU labeling (2.7 fold vs. control), while $\mathrm{Nb} /$ $\mathrm{NbL}$ cKO trabeculae exhibited a further increase in EdU labeling (6.2 fold vs. control) (Figure 2, A and $\mathrm{B})$. Cell cycle activity in the compact layer was unchanged between control and single or double cKOs (Figure 2B). Following these analyses, as double cKOs exhibited more severe phenotypes, we focused future analyses on double cKOs.

Additional quantitative proliferation analyses with a myocyte-specific cell cycle indicator, cyclin A2-EGFP (19), confirmed increased cell cycle activity in trabecular cardiomyocytes of $\mathrm{Nb} /$ $\mathrm{NbL}$ cKOs (Supplemental Figure 2, A-C). Furthermore, the cell cycle inhibitor KIP2/p57 was expressed in control trabeculae, but not in mutant trabeculae (Supplemental Figure 2D). The selective effect on trabecular proliferation in $\mathrm{Nb} / \mathrm{NbL}$ cKOs was consistent with higher levels of NUMB protein expression observed in trabeculae compared with the compact layer (Figure 2C). Quantitative analysis revealed 14-fold enrichment of $\mathrm{Nb}$ mRNA in trabeculae relative to compact layer (Supplemental Figure 2, E and F). 
A
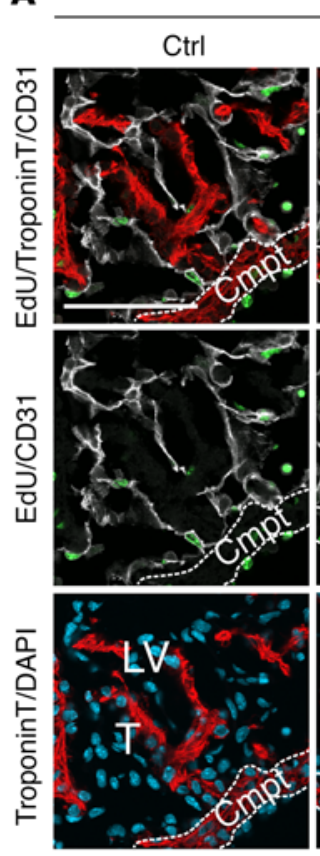

C
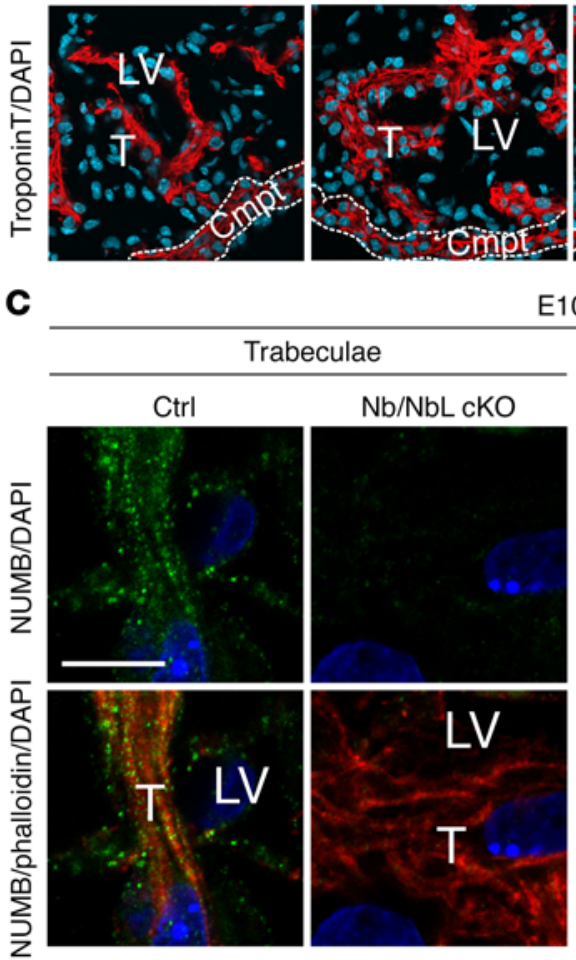

$\mathrm{Nb} / \mathrm{NbL} \mathrm{cKO}$
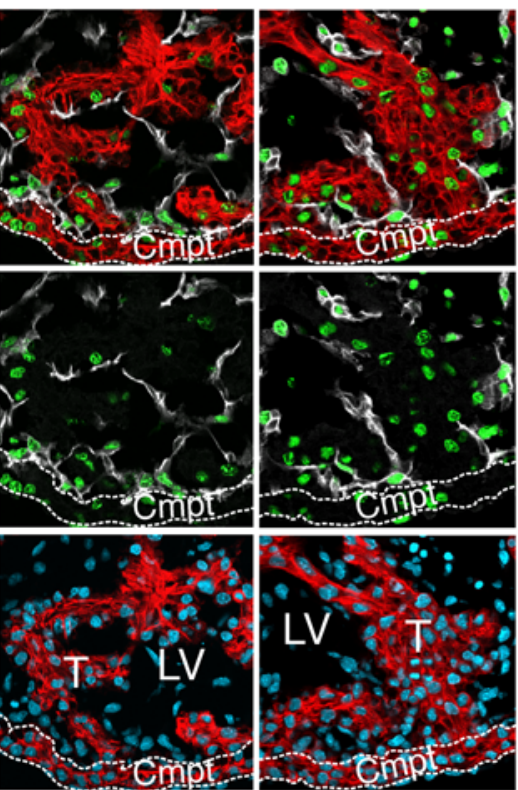

B

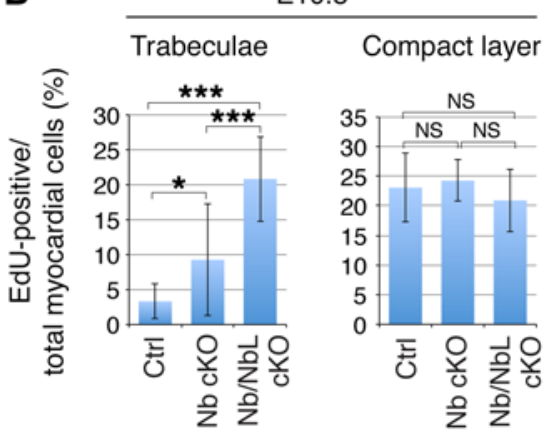

Aberrant activation of YAP1 and STAT5 in Nb/Nbl mutants. To identify factors driving abnormal trabecular proliferation, we undertook a candidate approach. Transcriptional cofactor and protooncogene YAP1, activated upon nuclear translocation, can induce proliferation in terminally differentiated cardiomyocytes $(20,21)$. We found that YAP1 was localized to significantly more trabecular nuclei in $\mathrm{Nb} / \mathrm{NbL}$ cKOs than in controls (Figure $3 \mathrm{~A}$ ).

Loss of $\mathrm{Nb} / \mathrm{Nbl}$ in skeletal muscle stem cells results in senescence (22); thus, mutant senescent cells could have a non-cell autonomous effect on proliferation of neighboring cells. To address whether increased proliferation of $\mathrm{Nb} / \mathrm{NbL} \mathrm{cKO}$ mutant trabecular cells was cell autonomous, we ablated $\mathrm{Nb} / \mathrm{Nbl}$ with doxycycline-inducible TnT-Cre (23), utilizing a doxycycline dose that resulted in mosaic Cre expression, as evidenced by R26-mTmG membrane EGFP expression (16). As shown in Supplemental Figure 3A, trabecular myocytes labeled by membrane EGFP (Nb/NbL mutant) exhibited nuclear localization of YAP1, whereas neighboring cells labeled by membrane tdTomato (Nb/NbL WT) did not exhibit nuclear YAP1. Thus, YAP1 nuclear localization and consequent increased proliferation were autonomous to $\mathrm{Nb} / \mathrm{NbL}$ mutant trabeculae.

Several signaling pathways can activate YAP1 (24). Hippo pathway kinases regulate cytoplasmic localization of YAP1 by phosphorylation at serine 112 in mice (25). Western blot analyses of whole heart extracts demonstrated no significant alteration in serine 112 phosphorylation or in total YAP1 protein (Figure 3B; see complete unedited blots in the supplemental material.). Although interpretation of Western blot data was tempered by the fact that these data were obtained from whole heart extracts, where trabeculae constitute a minor fraction of total cells, results suggested that pathways other than the Hippo pathway might be acting upstream of YAP1 in Nb/NbL mutant trabeculae. Therefore, other candidate signaling pathways were examined by quantitative Western blot analyses of E10.5 and E12.5 heart extracts (Figure 2C and Supplemental Figure 3B). In addition to ERK and Akt signal- 
A
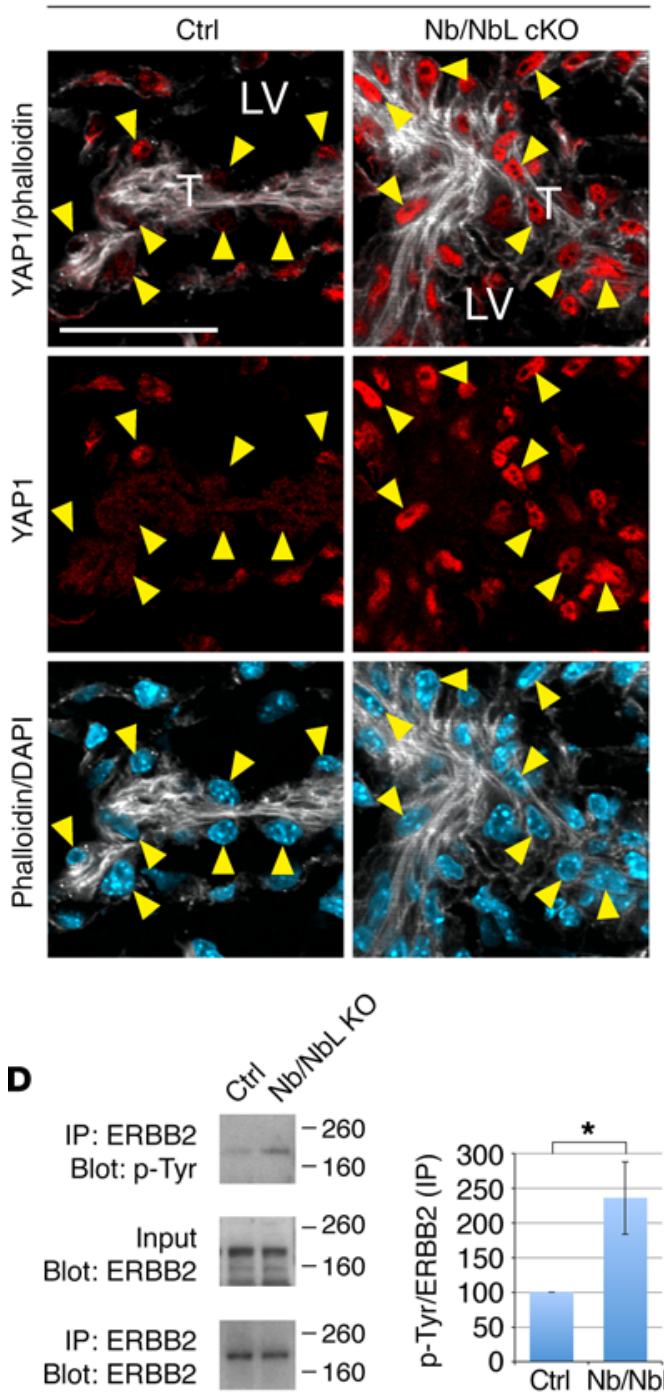
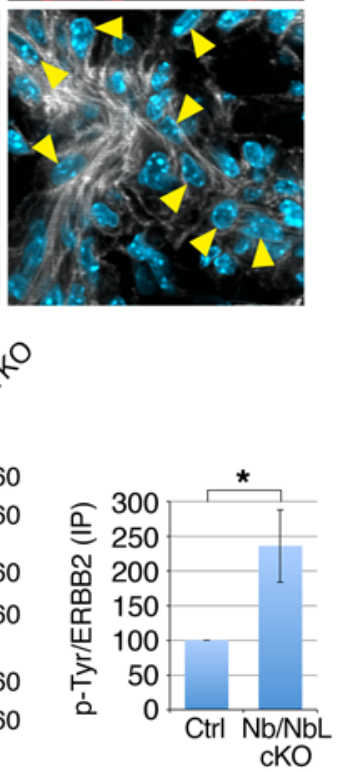

$\mathrm{Nb} / \mathrm{NbL}$ cKO

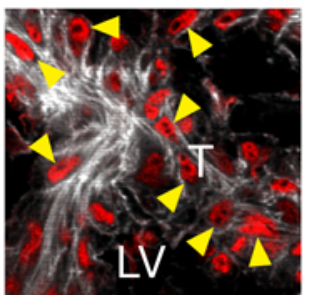

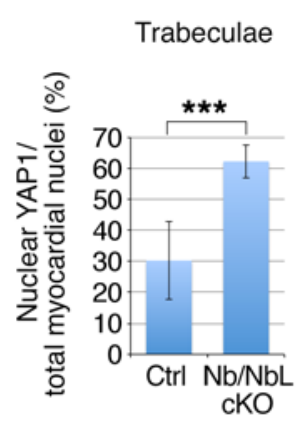
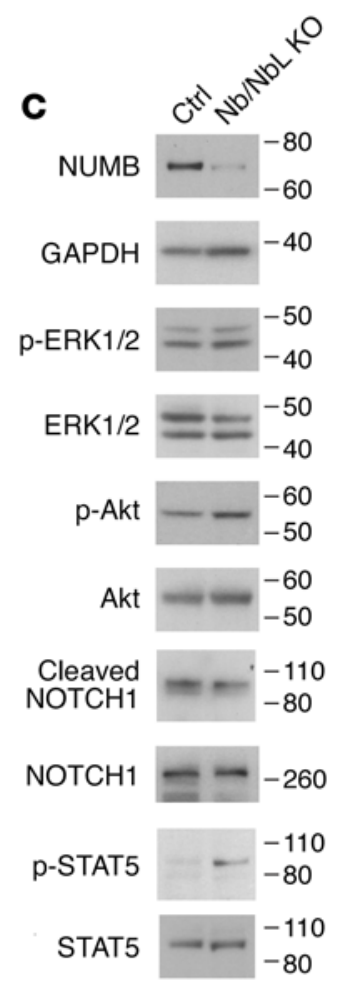

B

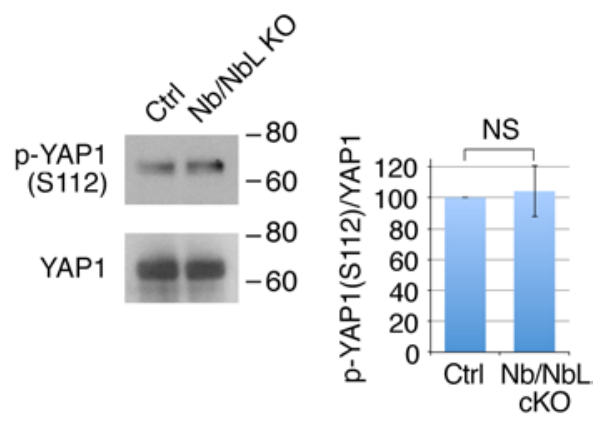

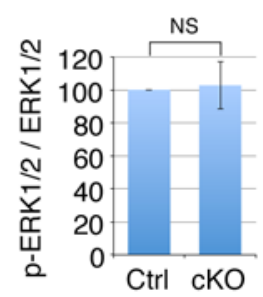

E E10.5
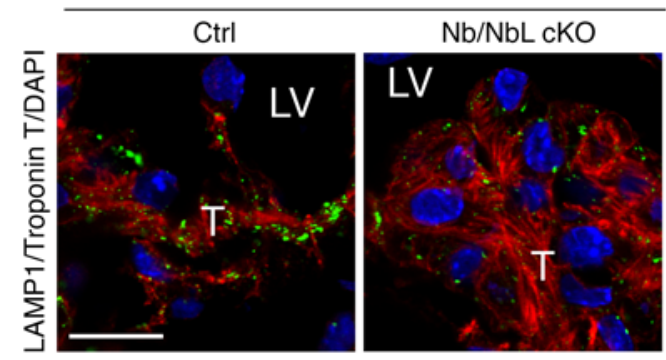

$\mathbf{F}$

\section{LAMP1}

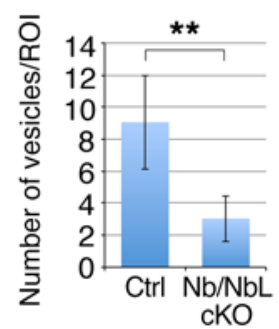

Figure 3. YAP1, STAT5, and ERBB2 are activated in Nb/NbL cKOs. (A) Immunofluorescence microscopy of E10.5 heart sections stained for DAPI (blue) and phalloidin (gray) and immunostained for YAP1 (red). Yellow triangles, trabecular nuclei. Scale bar: $50 \mu \mathrm{m}$. Quantitation revealed increased nuclear YAP1 in trabeculae of $\mathrm{Nb} / \mathrm{NbL}$ cKOs (control, $n=4$ mice; cKO, $n=4$ mice; 5 sections each). (B) Western blot analysis for YAP1 and p(S112)YAP1 of E10.5 heart extracts. Quantitation revealed no significant alteration in total YAP1 protein or p(S112)YAP1 in Nb/NbL cKOs when compared with controls (control, $n=3 ; c K O, n=3$ mice). (C) Western blot analysis for candidate signaling pathways from E10.5 heart extracts. Of candidate pathways examined, quantitative analyses revealed that only pSTAT5 was significantly increased in $\mathrm{Nb} / \mathrm{NbL}$ cKOs hearts when compared with controls. No alterations were observed in total NOTCH1 or NOTCH1 ICD (control, $n=3$ mice; CKO, $n=3$ mice). (D) Immunoprecipitation of E14.5 heart extracts with antibody to ERBB2 protein, followed by Western blot analysis for ERBB2 or phospho-tyrosine. Quantitation revealed increased activation/phosphorylation of ERBB2 in Nb/NbL cKOs relative to controls (control, $n=10$; cKO, $n=10$ mice; 3 to 4 hearts were pooled for 1 immunoprecipitation). (E) Immunofluorescence microscopy for LAMP1-positive LEs in trabeculae of control and Nb/NbL cKOs at E10.5. Scale bar: $20 \mu \mathrm{m}$. (F) Quantitative analysis revealed significantly reduced numbers of LAMP1-positive LEs in Nb/NbL cKO trabeculae relative to controls (control, $n=3$ mice; cKO, $n=3$ mice; 4 sections each). Data represent mean \pm SD. ${ }^{*} P<0.05 ;{ }^{* *} P<0.001 ;{ }^{* *} P<0.0001$, unpaired 2-tailed Student's $t$ test. 
A SiRNA
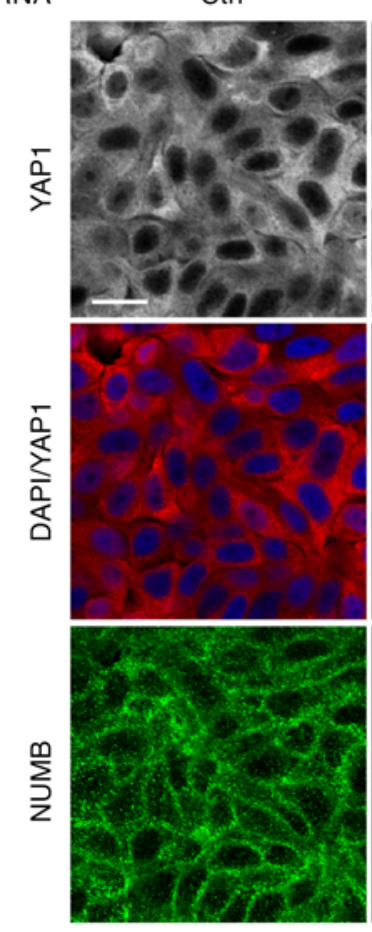

$\mathrm{Nb} / \mathrm{NbL}$

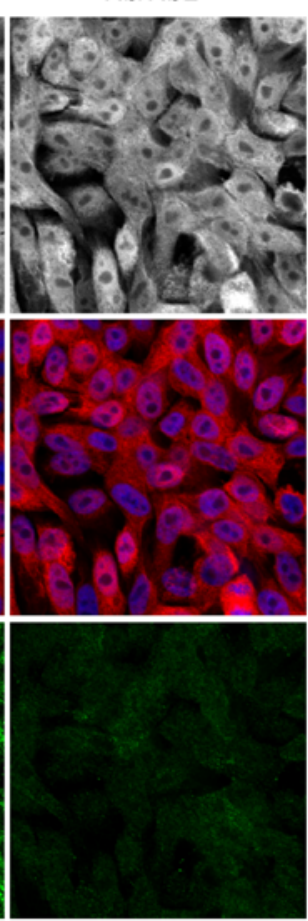

$\mathrm{Nb} / \mathrm{NbL}$ ERBB2
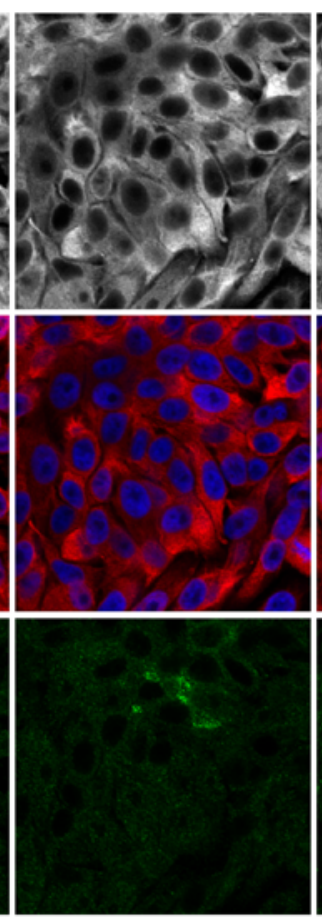

$\mathrm{Nb} / \mathrm{NbL}$ ERBB4
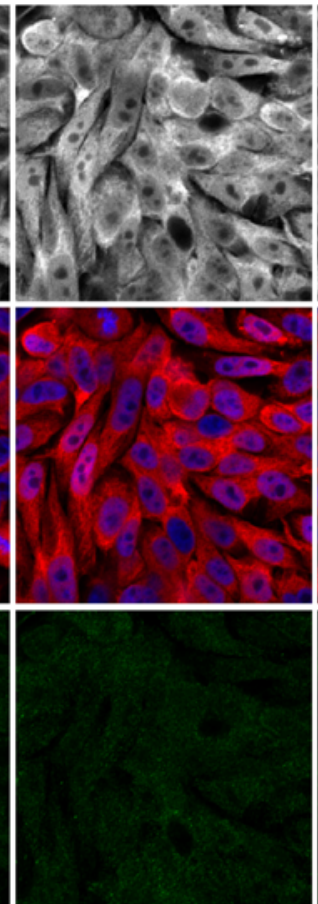

$\mathrm{Nb} / \mathrm{NbL}$ Stat5 inh

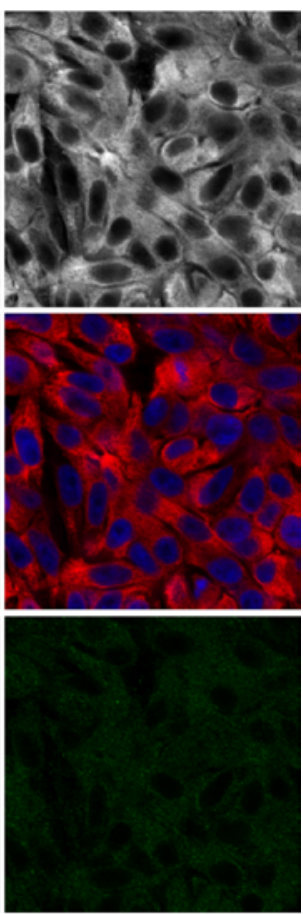

B

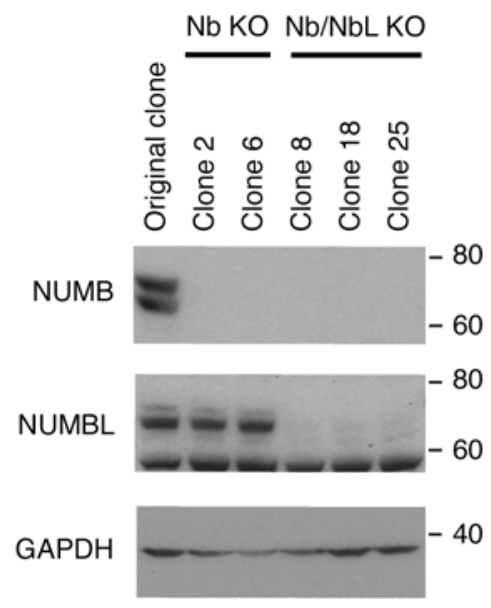

C

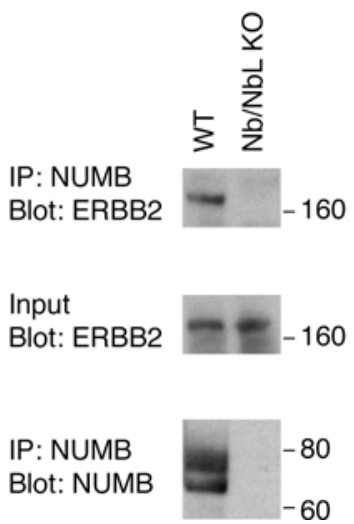

Figure 4. Aberrant YAP1 nuclear localization under confluent conditions in $\mathrm{Nb} / \mathrm{NbL}$ knockdown HeLa cells requires ERBB2 and STAT5. (A) Immunofluorescence microscopy of HeLa cells stained for DAPI (blue), YAP1 (gray or red), or NUMB (green). siRNAs that were utilized are indicated at the top of each panel. The STAT5 inhibitor nicotinoyl hydrazine was added 3 hours before fixation. Aberrant nuclear localization of YAP1 was prevented by siRNA knockdown of ERBB2 or by STAT5 inhibition, but not by siRNA knockdown of ERBB4. Scale bar: $20 \mu \mathrm{m}$. (B) Western blot analysis for NUMB and NUMBL in Nb KO or Nb/NbL KO HeLa cells. (C) Immunoprecipitation with antibody to NUMB in extracts from WT and Nb/NbL KO HeLa cells, followed by Western blot analysis with antibodies to ERBB2 or NUMB. ERBB2 interaction with endogenous NUMB was observed only in cells that were WT for NUMB, not in Nb/NbL KO cells. ing pathways, we examined STAT activation, as STAT is known to promote proliferation in other contexts. Of these pathways, phosphorylated STAT5 (pSTAT5) was significantly increased in Nb/ NbL cKOs (Figure 3C and Supplemental Figure 3B), suggesting it might be upstream of aberrant YAP1 nuclear localization.

In contrast to findings reported in a previous study (18), NOTCH1 intracellular domain (ICD) was not increased in Nb/NbL cKOs (Figure 3C and Supplemental Figure 3B). Utilizing an EGFP indicator for Notch transcriptional activation, transgenic Notch reporter (TNR) (26), no ectopic activation of Notch signaling was evident in mutant trabeculae, although the expected Notch-EGFP signal was evident in endocardium (Supplemental Figure 3C) (27). Since activation of NOTCH1 and NOTCH2 converges on the same transcriptional readout (28), this analysis suggested that neither NOTCH1 nor NOTCH2 was hyperactivated in $\mathrm{Nb} / \mathrm{NbL}$ cKO hearts at times when aberrant proliferation was evident.
ERBB2 as cargo for $N U M B$ and NUMBL. Phosphorylation of STAT5 occurs downstream of receptor tyrosine kinases (RTKs), including ERBB receptors (29-31). Ablation of Erbb2 or Erbb4 in mice is embryonic lethal owing to defects in myocardial proliferation and trabeculation $(2,3)$. Conversely, ERBB activation can result in aberrant myocyte proliferation (32). We hypothesized that aberrant STAT5 activation might reflect aberrant ERBB2/ERBB4 activation. Owing to lack of suitable antibodies for ERBB4, we first investigated ERBB2 activation, performing immunoprecipitation with anti-ERBB2 antibody in embryonic heart extracts, followed by quantitative Western blot analyses. As shown in Figure 3D, levels of tyrosine pERBB2 were significantly increased in $\mathrm{Nb} / \mathrm{NbL}$ cKOs relative to controls.

As NUMB and NUMBL are cargo-specific endocytic adaptor proteins (5), we performed protein interaction studies to investigate ERBB2 or ERBB4 as potential cargoes of NUMB or NUMBL. 
A
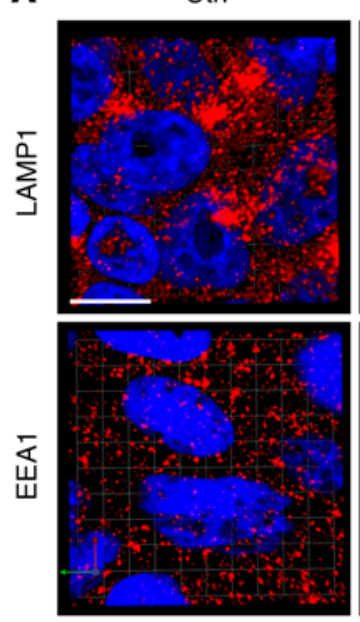

C

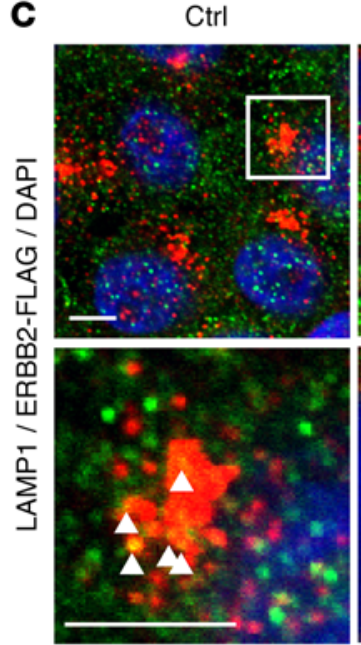

E

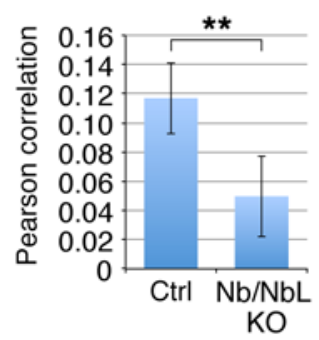

$\mathrm{Nb} / \mathrm{NbL} \mathrm{KO}$
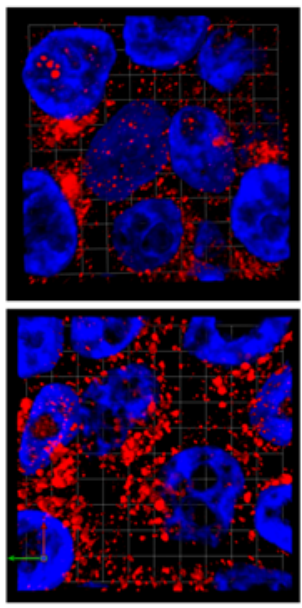

$\mathrm{Nb} / \mathrm{NbL} \mathrm{KO}$
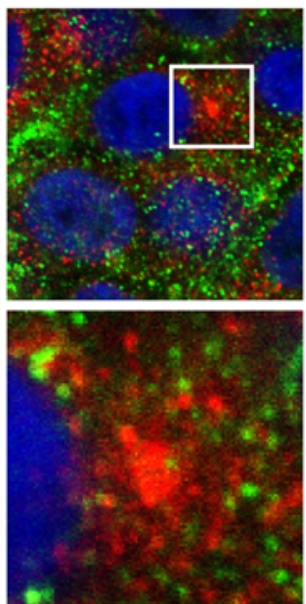

EEA1/ERBB2

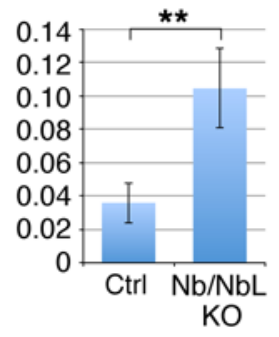

$\mathbf{B}$
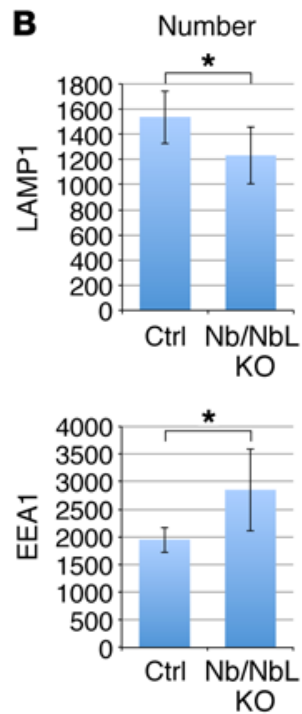

D

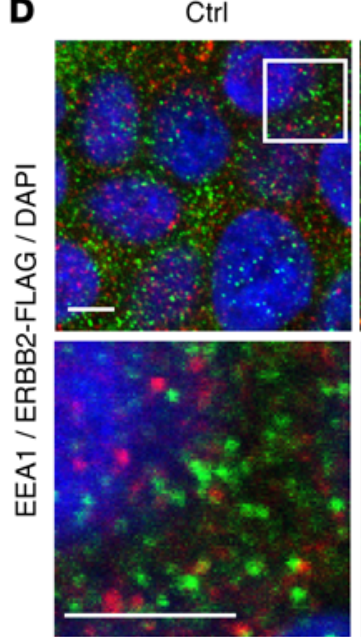

Sum volume $\left(\mu \mathrm{m}^{3}\right)$
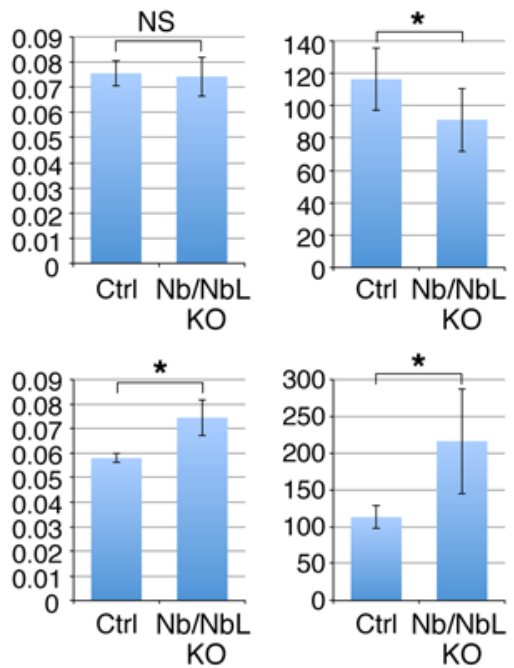

$\mathrm{Nb} / \mathrm{NbL} \mathrm{KO}$

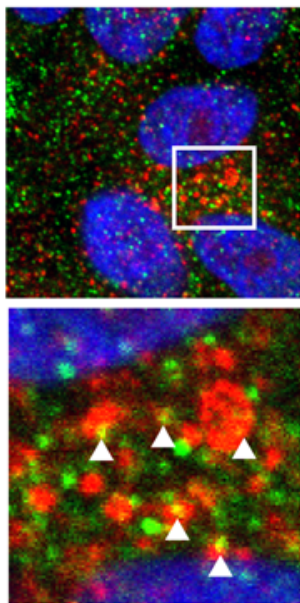

Figure 5. Partial block between EE and LE in Nb/NbL KO HeLa cells. (A) Immunofluorescence microscopy for markers of LE (LAMP1) and EE (EEA1) in control and $\mathrm{Nb} / \mathrm{NbL} \mathrm{KO}$ HeLa cells. Images shown are 3D reconstructions of Z-stack images. Scale bar: $10 \mu \mathrm{m}$. (B) Quantitation of LAMP1-positive LEs and EEA1-positive EEs ( $n=3,6$ visual fields each). (C and $\mathbf{D}$ ) Immunofluorescence microscopy to examine colocalization of ERBB2 (green) with LAMP1 (red) (C) or colocalization of ERBB2 (green) with EEA1 (red) (D) in control and Nb/NbL KO HeLa cells. Bottom panels are magnified images of square areas in top panels. Scale bars: $5 \mu \mathrm{m}$. (E) Quantitation of colocalization of ERBB2 and LAMP1 or EEA1 ( $n=3,6$ visual fields each). Data represent mean $\pm \mathrm{SD}$. ${ }^{*} P<0.05$; ${ }^{*} P<0.001$, 2-tailed Student's $t$ test.

Coimmunoprecipitation studies were performed in extracts of 293T cell transiently overexpressing relevant tagged proteins (Supplemental Figure 3, D and E). Results demonstrated that ERBB2 and ERBB4 interacted with all 4 isoforms of NUMB, but that only ERBB2 specifically interacted with NUMBL (Supplemental Figure 3E). As NUMBL plays a redundant role with NUMB in trabeculae, this suggested that ERBB2 might be a more relevant cargo than ERBB4 in this context.

To determine the specificity of the interaction between NUMB and ERBB2, we generated deletion mutants of $\mathrm{Nb}$ and Erbb2 (Supplemental Figure 4A). Since p65/p66 isoforms of NUMB were expressed in embryonic heart by reverse-transcription PCR (RT-PCR) and Western blot (Supplemental Figure 3D, F, and G; and Supplemental Table 1), we utilized p65 NUMB. Numb finger domain (NUMBF) and proline-rich regions of NUMB specifically interacted with ERBB2, and the C-terminal domain of ERBB2 specifically interacted with NUMB (Supplemental Figure 4, B and C).

If ERBB2 were a specific cargo for NUMB and NUMBL, intracellular targeting of ERBB2 might be perturbed in Nb/NbL KOs. RTKs are endocytosed from plasma membrane into early endosomes (EEs) and can be recycled or targeted for lysosomal degradation through late endosomes (LEs) (33). To investigate potential perturbations in 
A

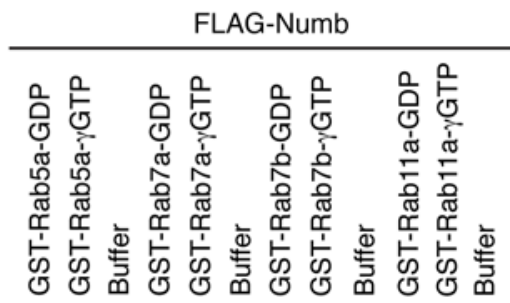

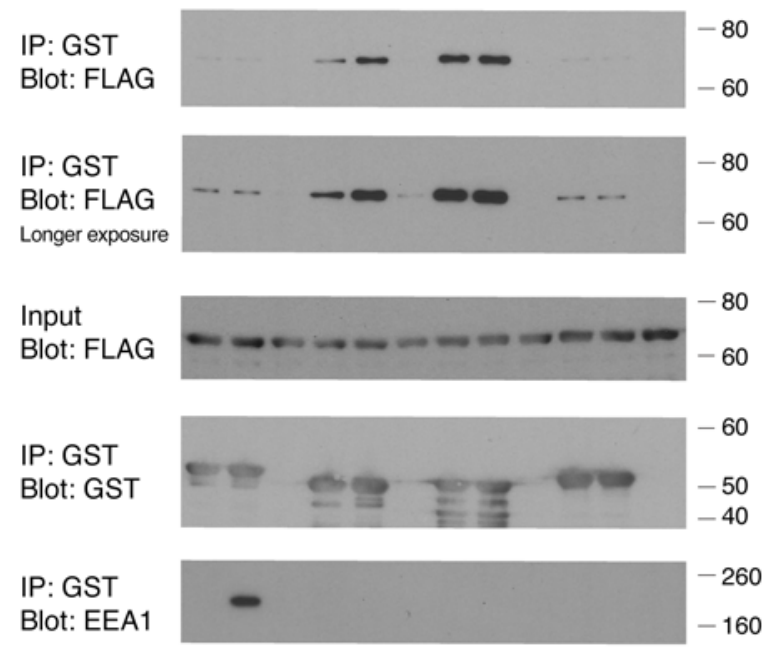

C
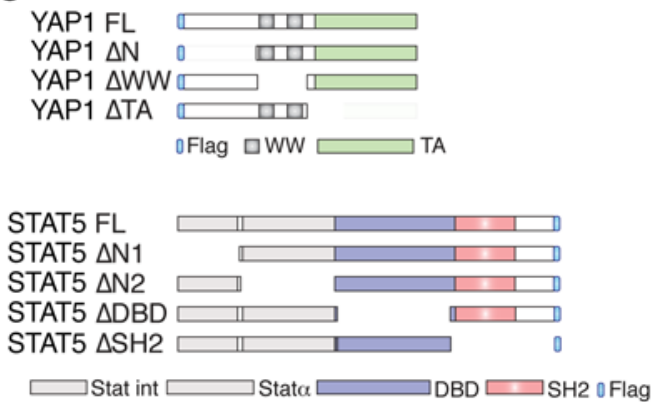

E

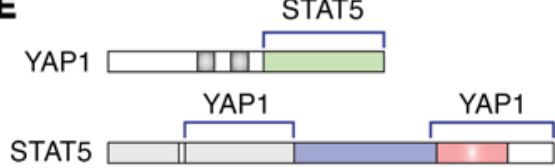

B
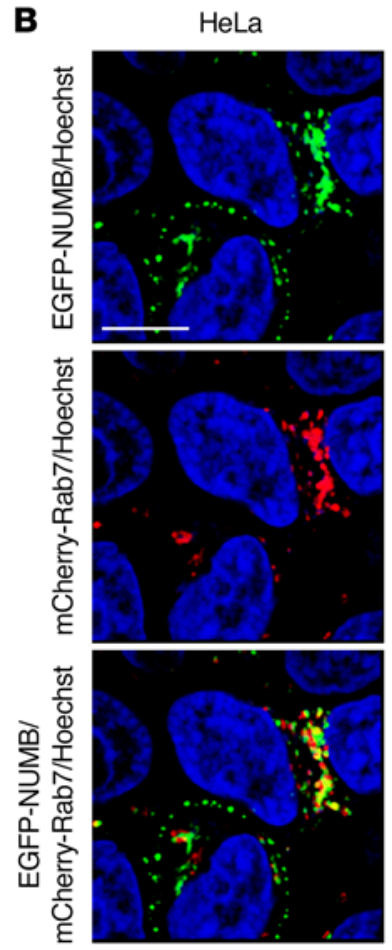

D
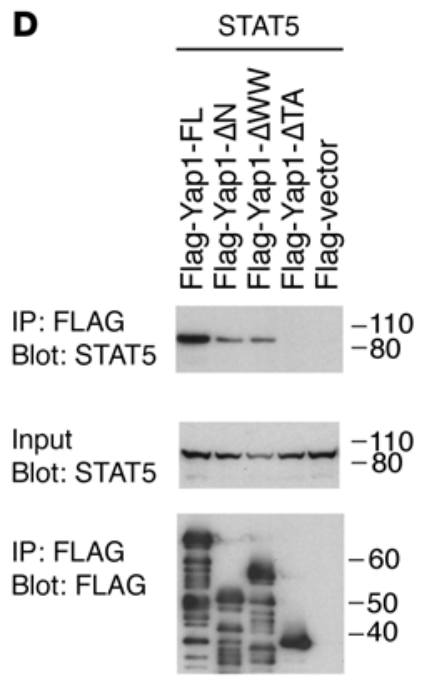

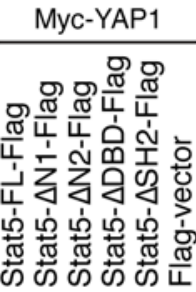

IP: FLAG

Blot: Myc

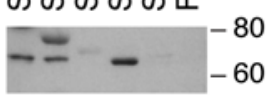

Input

Blot: Myc

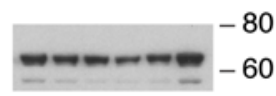

IP: FLAG

Blot: FLAG

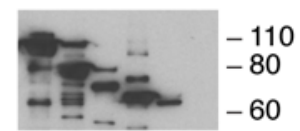

Figure 6. NUMB and NUMBL interact with Rab7, and YAP1 interacts with STAT5. (A) Interaction of FLAG-tagged NUMB with GST-tagged GDP- or GTPbound Rabs. As expected, only the active GTPYS-bound form of Rab5a interacted with EEA1 (bottom panel), indicating that the addition of either GDP or GTP $\gamma S$ to Rab proteins was successful. NUMB interaction with Rab7a or Rab7b was readily detectable, whereas interactions between NUMB and Rab5a or Rab11a were detectable only upon prolonged exposure. Note preferential association of NUMB with the active GTP $\gamma$ S-bound form of Rab7a. (B) Live images of HeLa cells stably expressing EGFP-NUMB and mCherry-Rab7a with nuclear Hoechst 33342 staining. NUMB and Rab7a were strongly colocalized. Scale bar: $10 \mu \mathrm{m}$. (C) FLAG-tagged deletion constructs of Yap1 and Stat5. (D) Immunoprecipitation of FLAG-tagged Yap1 deletion constructs from cotransfected 293 T cell extracts was followed by Western blot analysis with antibody to STAT5. Conversely, immunoprecipitation of FLAG-tagged Stat5 deletion constructs from cotransfected $293 \mathrm{~T}$ cell extracts was followed by Western blot analysis with antibody to Myc. (E) Domains within YAP1 and STAT5 required for their interaction.

vesicular trafficking in $\mathrm{Nb} / \mathrm{NbL}$ cKOs, we examined LEs by immunostaining with anti-LAMP1 antibody (34). The number of LAMP1-positive LEs was significantly reduced in mutant trabeculae (Figure 3, E and F, and Supplemental Figure 4D), while Western blot analyses of whole heart extracts demonstrated no significant alterations in total LAMP1 protein in mutant hearts relative to controls (Supplemental Figure $4 \mathrm{E}$ ). Western blot data, however, should be interpreted with the consideration that LAMP1 protein in trabecular myocytes represents a minor fraction of LAMP1 protein in whole heart.

YAP1 activation consequent to loss of $\mathrm{Nb} / \mathrm{Nbl}$ requires $\mathrm{ERBB} 2$ and STAT5. To investigate whether activation of YAP1 in Nb/ NbL cKOs was dependent on ERBB2 and/or STAT5 activation, we established a HeLa cell system. To facilitate visualization of ERBB2, we generated a transgenic HeLa cell line expressing FLAG-tagged 
A

E10.5

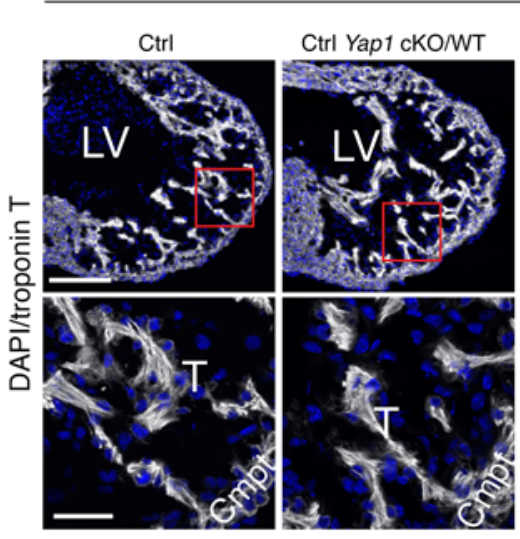

Ctrl Erbb2 AWT
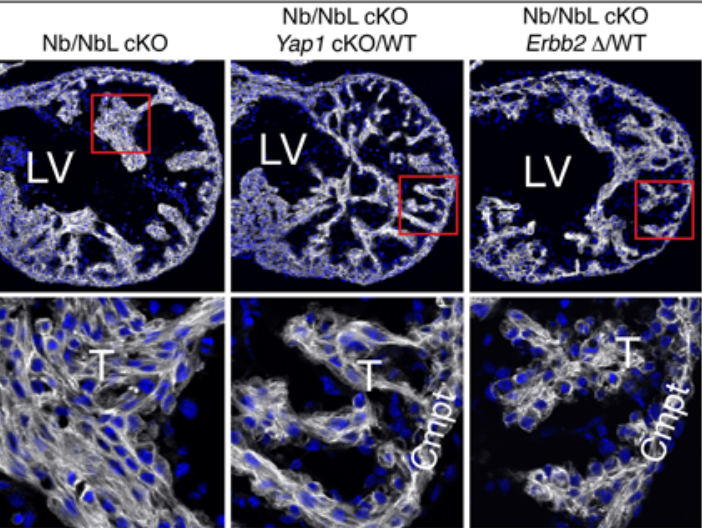

B

Trabeculae

Compact layer
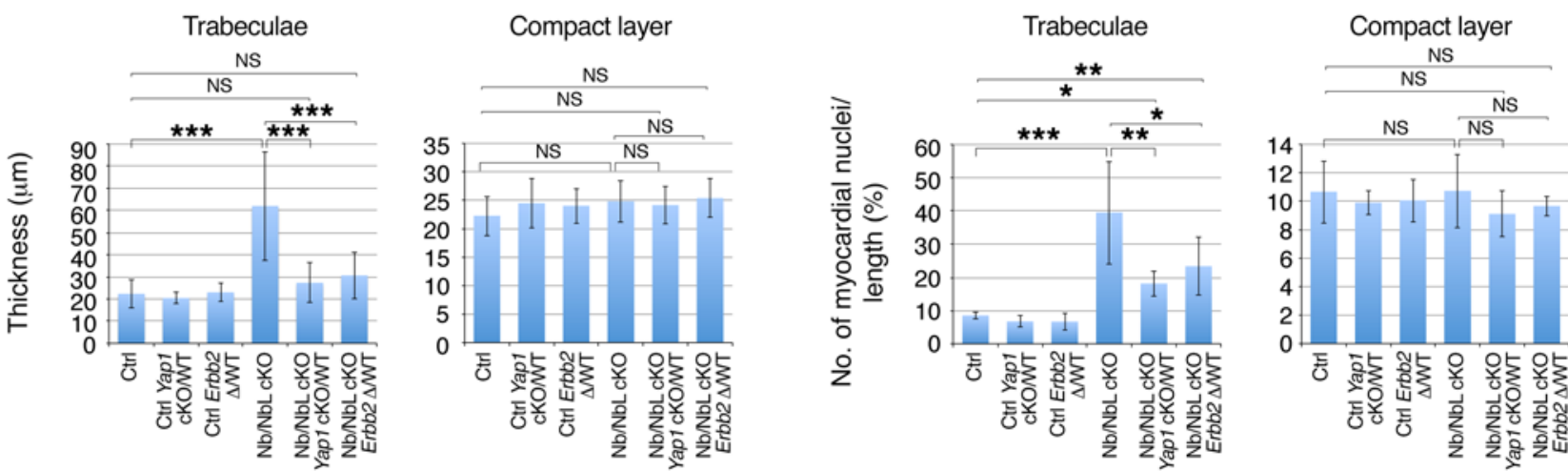

C

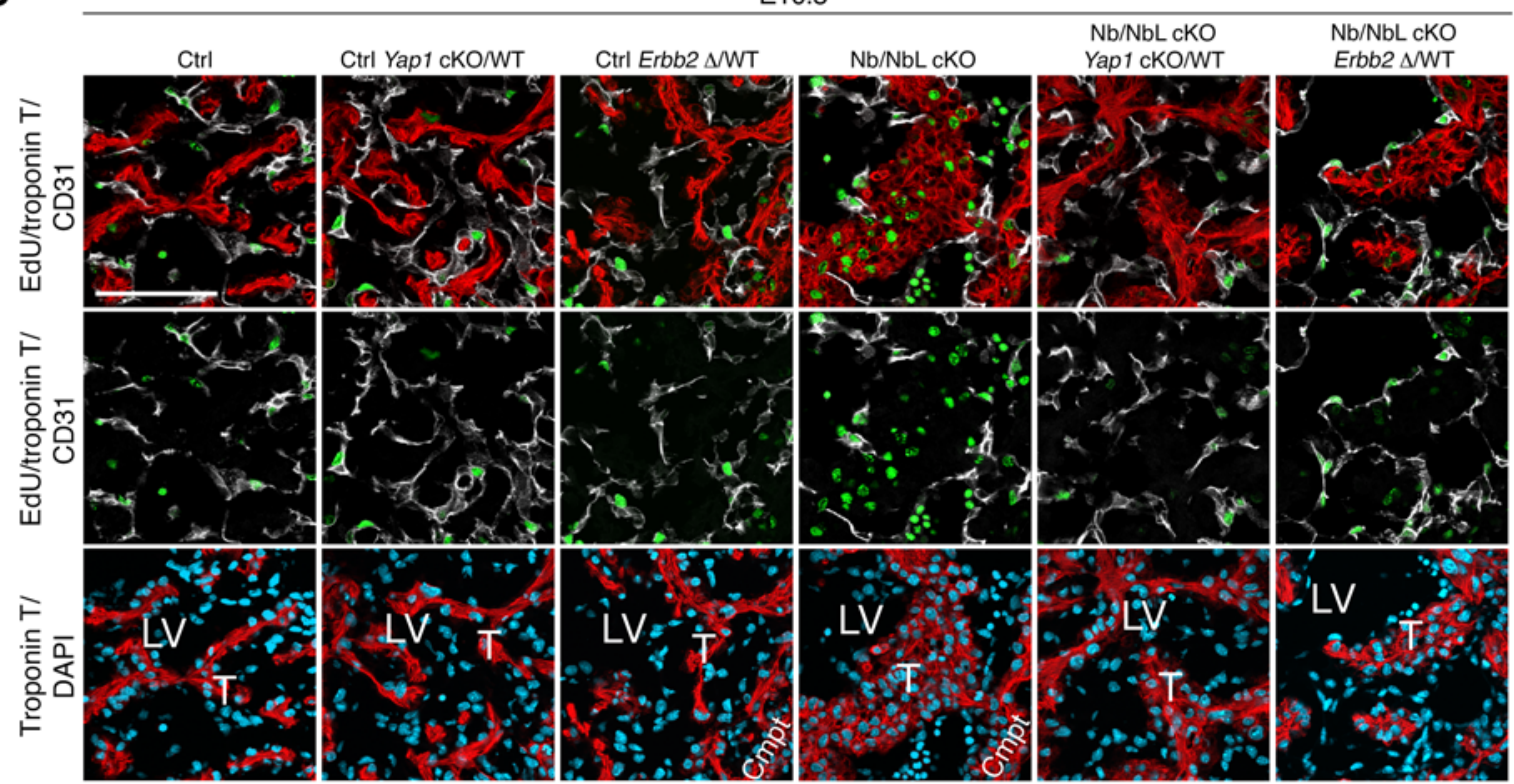

D

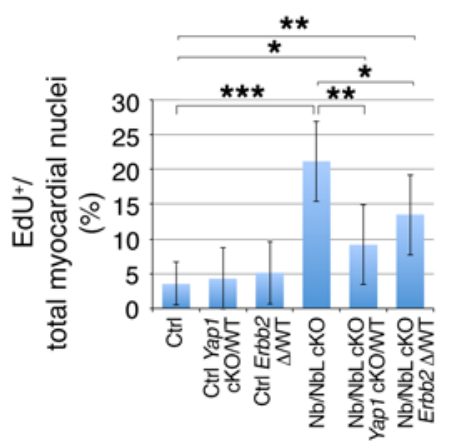

Compact layer

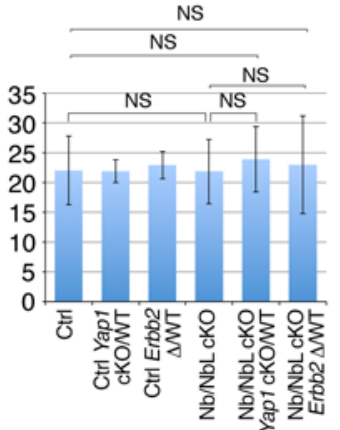

Figure 7. Heterozygosity for either Yap1 or Erbb2 significantly rescues Nb/NbL cKO phenotypes. (A) Immunofluorescence microscopy of E10.5 heart sections stained with DAPI (blue) and antibody to troponin T (gray). Heterozygosity for Yap1 or Erbb2 in Nb/NbL cKO backgrounds significantly rescues abnormal trabecular phenotypes, as quantitated in B. Scale bars: $200 \mu \mathrm{m}$ (top panels); 40 $\mu \mathrm{m}$ (bottom panels). (B) Quantitative analyses $(n=4, n=2, n=2, n=4, n=3$, and $n=3$ mice each group, 6 sections each). (C) Immunofluorescence microscopy of EdU-labeled E10.5 trabeculae within left ventricle sections, stained with DAPI (blue), and with antibodies to TnT (red; trabeculae) and CD31 (gray, endocardium). Scale bar: $100 \mu \mathrm{m}$. (D) Quantitative analyses $(n=4, n=2, n=2, n=4, n=3$, and $n=3$ mice each group, 6 sections each). Data represent mean $\pm S D$. ${ }^{*} P<0.05$; ${ }^{* *} P<0.001 ;{ }^{* *} P<0.0001$, 1-way ANOVA followed by Tukey's post hoc test. 
A

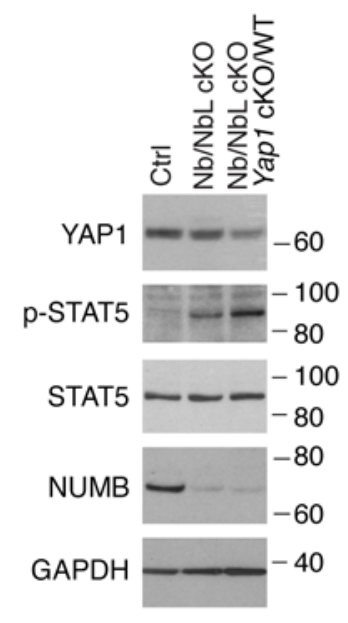

B

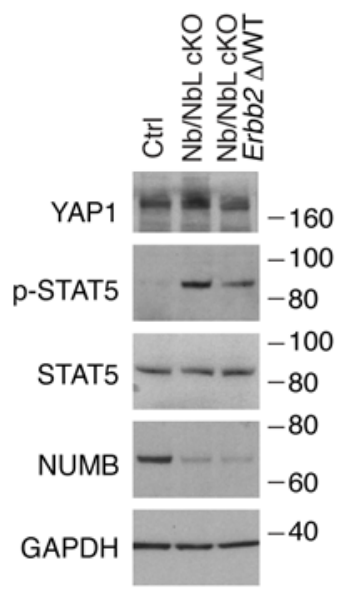

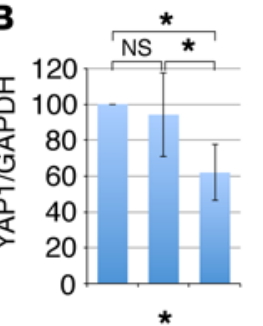
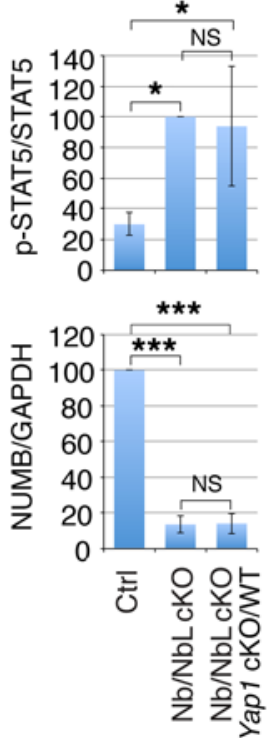
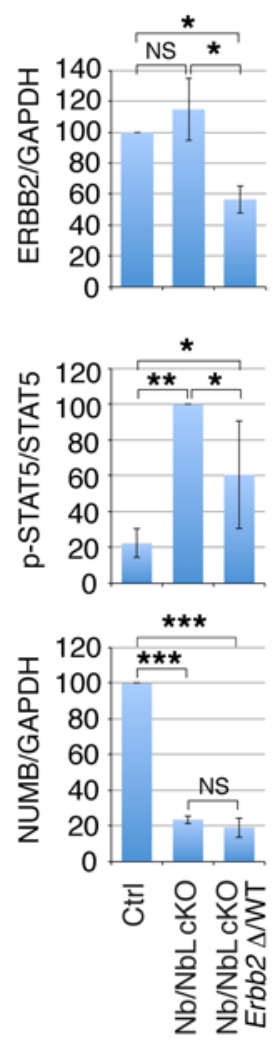

Figure 8. Increased pSTAT5 in Nb/NbL cKO was dependent on ERBB2. (A) Western blot analysis for YAP1, pSTAT5, STAT5, NUMB, and GAPDH of E10.5 heart extracts from control, Nb/NbL cKOs, and Nb/NbL cKOs heterozygous null for Yap1 or Erbb2, with quantitative analyses shown in B. Data demonstrated that Nb/NbL cKOs that were heterozygous for Yap1 exhibited decreased YAP1 and still exhibited loss of NUMB and NUMBL to an extent comparable to that of Nb/NbL cKOs. Nb/NbL cKOs that were heterozygous for Erbb2 had reduced pSTAT5 relative to Nb/NbL cKOs, but exhibited loss of NUMB and NUMBL to an extent comparable to that observed in Nb/NbL cKOs ( $n=3$ mice each group). Data represent mean \pm SD. ${ }^{*} P<0.05 ;{ }^{* *} P<0.001 ;{ }^{* *} P<0.0001,1$-way ANOVA followed by Tukey's post hoc test.

ERBB2 (Supplemental Figure 5A). This cell line demonstrated the expected response of YAP1 to sparse (nuclear YAP1) or confluent (cytoplasmic YAP1) conditions $(25,35)$. We then performed siRNA knockdown of NUMB and NUMBL (hereafter referred to as Nb/ $\mathrm{NbL}$ knockdown) and investigated YAP1 localization under confluent conditions, where it is normally cytoplasmic (Supplemental Table 2). Consistent with our in vivo findings, loss of NUMB and NUMBL resulted in aberrant YAP1 nuclear localization (Figure 4A).

Next, we performed siRNA knockdown of ERBB2 or ERBB4 in $\mathrm{Nb} / \mathrm{NbL}$ knockdown cells. Knockdown of ERBB2 abrogated effects of $\mathrm{Nb} / \mathrm{NbL}$ knockdown, resulting in cytoplasmic YAP1 localization (Figure 4A). In contrast, knockdown of ERBB4 did not override effects of $\mathrm{Nb} / \mathrm{NbL}$ knockdown, as YAP1 remained localized to the nucleus (Figure 4A). Addition of a specific STAT5 inhibitor also abrogated effects of $\mathrm{Nb} / \mathrm{NbL}$ knockdown on YAP1 nuclear localization (Figure 4A). Thus, YAP1 nuclear translocation consequent to $\mathrm{Nb} / \mathrm{NbL}$ knockdown was mediated by ERBB2 and STAT5.

In these knockdown experiments, NUMB protein was significantly diminished (Figure 4A), and knockdown efficiencies with NUMB, NUMBL, ERBB2, or ERBB4 siRNAs were approximately 90\% at the transcript level (Supplemental Figure 5B and Supplemental Table 3). However, due to concerns of potential off-target effects (Supplemental Figure 5B) and incomplete knockdown, we generated $\mathrm{Nb} / \mathrm{Nbl}$ null (Nb/NbL KO) HeLa cells utilizing Crispr/
Cas9n (Supplemental Figure 5, C and D, and Supplemental Table 4) (36) and confirmed complete loss of NUMB and NUMBL in 3 independent HeLa cell clones (Figure 4B). YAP1 behavior in Nb/ $\mathrm{NbL}$ KO cell lines mirrored that observed in siRNA knockdown experiments (compare Figure 4A and Supplemental Figure 5E), reaffirming that YAP1 nuclear translocation consequent to $\mathrm{Nb}$ / Nbl loss was dependent on ERBB2 and STAT5.

YAP1 nuclear localization in confluent conditions in $\mathrm{Nb} / \mathrm{NbL}$ KO cells suggested that normal contact inhibition might be overridden in $\mathrm{Nb} / \mathrm{NbL} \mathrm{KO}$ cells. Indeed, proliferation assays demonstrated significantly increased proliferation of $\mathrm{Nb} / \mathrm{NbL} \mathrm{KO}$ cells relative to control cells under confluent conditions (Supplemental Figure 5F).

Our biochemical studies with transiently transfected expression constructs had demonstrated interaction between NUMB and ERBB2. To investigate NUMB interaction with ERBB2 at endogenous levels of NUMB protein, lysates from WT and Nb/ NbL KO HeLa cells were immunoprecipitated with anti-NUMB antibody and blotted with anti-ERBB2 antibody. As shown in Figure 4C, endogenous NUMB interaction with ERBB2 was evidenced in WT HeLa cells, but not in Nb/NbL KO cells. Although these results further demonstrated an interaction between NUMB/NUMBL and ERBB2, the possibility remains that the observed interaction between NUMB and ERBB2 is indirect, perhaps mediated by another protein. 
A

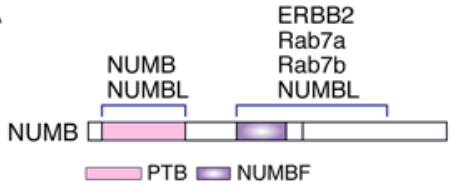

B The role of NUMB in cell cycle withdrawal

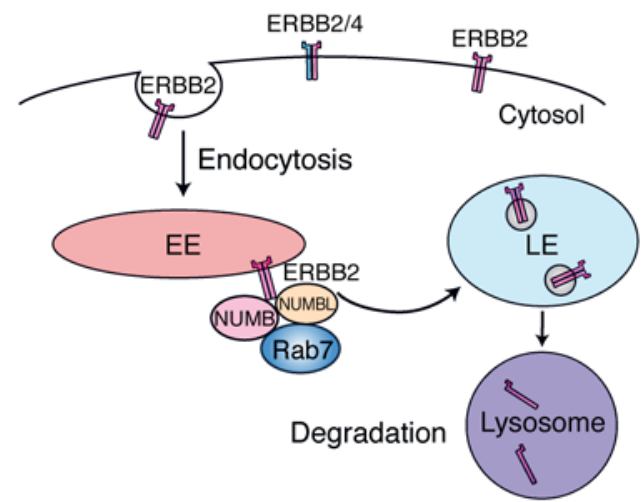

C Loss of NUMB leads to aberrant cell proliferation

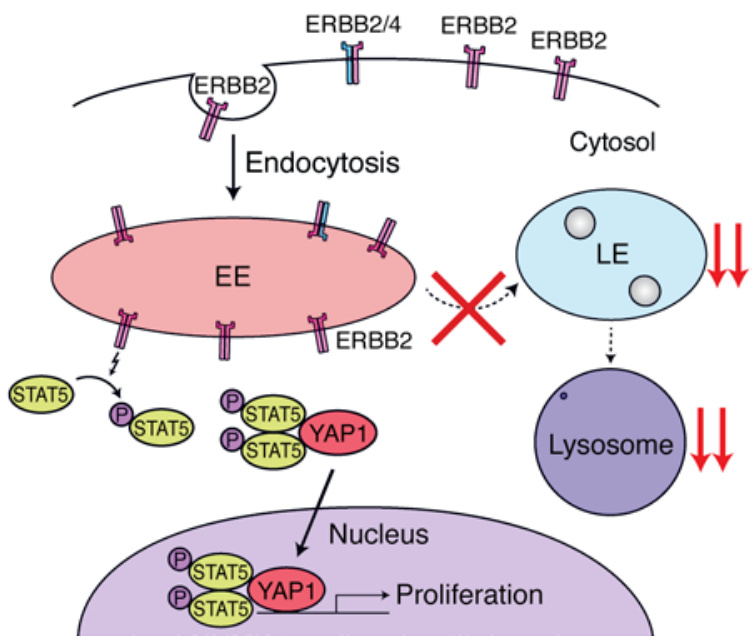

Figure 9. NUMB and NUMBL are multifaceted endocytic adaptors with key roles in cell cycle regulation. (A) Diagram of NUMB domains that interact with other proteins as identified in this study. (B) Model depicting the role of NUMB and NUMBL in cell cycle withdrawal. (C) Model depicting effects of NUMB and NUMBL loss on aberrant cell proliferation.

Partial block between EE and LE. Since we had observed reduced numbers of LEs in developing hearts of $\mathrm{Nb} / \mathrm{NbL}$ cKOs, we examined the number of LEs in our HeLa system. We also examined the number of EEs marked by EEA1 (37). Consistent with in vivo data, LEs were reduced in $\mathrm{Nb} / \mathrm{NbL}$ KO HeLa cells (Figure $5 \mathrm{~A}$ ). Examination of EEs demonstrated abnormally large aggregates in KO HeLa cells (Figure 5A). Quantitative analyses supported these findings (Figure 5B). These observations suggested a partial block to vesicle trafficking between the EE and LE consequent to loss of $\mathrm{Nb} / \mathrm{Nbl}$.

We further investigated colocalization of ERBB2 with EE or LE compartments. Colocalization of ERBB2 with LEs was significantly reduced in Nb/NbL KO cells (Figure 5, C and E). In contrast, colocalization of ERBB2 with EEs was significantly increased in
KO cells (Figure 5, D and E). Together, these results suggested that transit of ERBB2 from EE to LE was perturbed in KO cells.

The foregoing suggested that NUMB and NUMBL played a pivotal role in targeting ERBB2 cargo from EE to LE, but how NUMB and NUMBL effected this endosomal transition remained to be addressed. Small GTPase Rab proteins are critical regulators of endosomal transitions $(38,39)$. Given the role of Rab7 in the transition from EE to LE (40), we hypothesized that NUMB and NUMBL might specifically interact with Rab7 to facilitate cargo targeting from EEs to LEs.

To investigate interactions between NUMB and Rab proteins, we purified GST-tagged Rab5a, Rab7a, Rab7b, and Rab11a recombinant proteins (Supplemental Figure 6A), generating both inactive GDP-bound forms and constitutively active GTP $\gamma$ S-bound forms (41). Each GDP/GTP $\gamma$ S bound inactive/active form of Rab was mixed with 293T cell lysates overexpressing FLAG-tagged NUMB-p65. By GST pull-down, Rab5a, Rab7a, Rab7b, and Rab11a each interacted with NUMB, but the interaction with Rab7a and Rab7b was most significant (Figure 6A). Moreover, consistent with NUMB playing a positive role in the transition from EE to LE, NUMB preferentially interacted with the GTP-bound active form of Rab7a rather than the GDP-bound inactive form (Figure 6A). We next investigated which isoforms of NUMB preferentially interacted with Rab7a/Rab7b. Both Rab7a and Rab7b preferentially interacted with p71-NUMB, p65-NUMB, and NUMBL (Supplemental Figure 6B).

To further investigate the specificity of these interactions, we investigated domain-specific interactions between NUMB and $\mathrm{Rab7a} / \mathrm{Rab} 7 \mathrm{~b}$ and found that NUMBF and proline-rich regions of NUMB interacted with Rab7a/Rab7b (Supplemental Figure 6C). To validate interaction of Numb and Rab7 within a cellular context, HeLa cells stably expressing EGFP-tagged NUMB and mCherry-tagged Rab7a were generated. EGFP-tagged NUMB substantially colocalized with mCherry-tagged Rab7a (Figure 6B). Together, the foregoing results were consistent with NUMB and NUMBL assisting in transitioning ERBB2 cargo from EEs to LEs via specific interactions of NUMB and NUMBL with Rab7.

STAT5 can directly interact with YAP1. Loss of $\mathrm{Nb} / \mathrm{Nbl}$ caused aberrant activation of an ERBB2/STAT5 pathway, resulting in YAP1 nuclear localization. How aberrant activation of ERBB2/ STAT5 led to YAP1 nuclear translocation remained to be addressed. Because we had observed no decrease in Hippo-mediated YAP1 phosphorylation (serine 112) in $\mathrm{Nb} / \mathrm{NbL}$ cKOs, we hypothesized that STAT5 might interact with YAP1, thus retaining YAP1 in the nucleus, as pSTAT5 is retained in the nucleus $(29,42)$.

To investigate specific interactions between YAP1 and STAT5, we generated deletion mutants of YAP1 and STAT5 (Figure 6C). Interaction assays demonstrated that the transactivation domain of YAP1 was required for interaction with STAT5, while the SH2 domain of STAT5 was required for interaction with YAP1 (Figure $6, \mathrm{D}$ and E). These data indicated that interaction of STAT5 with YAP1 could result in nuclear retention of YAP1.

NUMB interacts with NUMBL. Because our studies demonstrated that both NUMB and NUMBL specifically interacted with ERBB2 and Rab7 to target ERBB2 to a degradative pathway, we wondered whether NUMB and NUMBL might also interact with each other. Indeed, NUMBL interacted with p65/p71 NUMB, while p65 NUMB interacted with NUMB itself, preferentially with the same isoform 
(Supplemental Figure 6D). NUMB interacted with NUMB itself through the phosphotyrosine-binding (PTB) domain and interacted with NUMBL through the PTB domain and the proline-rich region (Supplemental Figure 6E). These data suggested that NUMB might act as a homodimer or as a heterodimer with NUMBL.

Reduction of YAP1 or ERBB2 significantly rescues $\mathrm{Nb} / \mathrm{NbL}$ cKO trabecular phenotypes. In HeLa cells, loss of $\mathrm{Nb} / \mathrm{Nbl}$ caused aberrant nuclear localization of YAP1 in confluent conditions that was dependent on activation of an ERBB2/STAT5 pathway. To further substantiate this pathway as being causative for aberrant trabecular phenotypes in myocardial $\mathrm{Nb} / \mathrm{NbL}$ cKOs, we performed in vivo rescue studies. Both ERBB2 and YAP1 are required prior to E10 for cardiomyocyte proliferation $(2,43)$, preceding the requirement for NUMB and NUMBL in trabecular cell cycle withdrawal. Therefore, a "rescue" experiment with homozygous loss of function for these genes would be problematic. Accordingly, we performed rescue experiments with heterozygous loss-of-function alleles. Heterozygous floxed Yap1 or an Erbb2 null allele (44, 45) was introduced into $\mathrm{Nb} / \mathrm{NbL} \mathrm{cKO}$ backgrounds. Quantitative analyses revealed that the thickened trabecular phenotype of $\mathrm{Nb} / \mathrm{NbL}$ cKOs at E10.5 was significantly rescued by the presence of either the floxed Yap1 allele or the Erbb2 null allele, while these alleles did not affect the compact layer (Figure 7, A and B). Quantitative EdU analyses further demonstrated that heterozygosity for either Yap1 or Erbb2 significantly reduced aberrant trabecular proliferation in $\mathrm{Nb} / \mathrm{NbL}$ cKOs (Figure 7, C and D).

Quantitative Western blot analyses of E10.5 hearts (Figure 8, A and B) demonstrated that heterozygosity for Yap1 or Erbb2 resulted in significantly decreased YAP1 or ERBB2 protein levels in $\mathrm{Nb} / \mathrm{NbL}$ cKOs. Importantly, levels of NUMB were reduced to the same extent in the presence or absence of floxed Yap1 or null Erbb2 alleles in $\mathrm{Nb} / \mathrm{NbL}$ cKOs. Decreased levels of ERBB2 in $\mathrm{Nb} / \mathrm{NbL}$ cKOs resulted in a significant reduction $(P=0.030)$ in pSTAT5 levels relative to $\mathrm{Nb} / \mathrm{NbL} \mathrm{cKO}$, indicating that increased pSTAT5 was dependent on ERBB2. In contrast, decreased levels of YAP1 had no effect on pSTAT5 levels relative to pSTAT5 levels in $\mathrm{Nb} / \mathrm{NbL}$ cKOs, consistent with pSTAT5 acting upstream of YAP1. Together, these in vivo rescue studies demonstrated that trabecular phenotypes of $\mathrm{Nb} / \mathrm{NbL}$ cKOs could be substantially rescued by loss of function for either ERBB2 or YAP1 and demonstrated the dependence of observed phenotypes on an ERBB2/STAT5/YAP1 pathway.

\section{Discussion}

Our in vivo analysis of cardiomyocyte-specific $\mathrm{Nb} / \mathrm{NbL}$ cKO mice revealed increased proliferation and aberrant activation of an ERBB2/ STAT5/YAP1 pathway in mutant trabeculae that was accompanied by decreased numbers of LEs. Consistent with these in vivo findings, studies in $\mathrm{Nb} / \mathrm{NbL}$ knockdown or KO HeLa cells revealed increased proliferation and aberrant nuclear localization of YAP1 under confluent conditions and decreased numbers of LEs. Aberrant YAP1 nuclear localization in $\mathrm{Nb} / \mathrm{NbL} \mathrm{KO}$ HeLa cells was dependent on ERBB2 and STAT5. In mouse embryos, heterozygous loss of function for ERBB2 or YAP1 demonstrated substantial rescue of trabecular phenotypes in $\mathrm{Nb} / \mathrm{NbL}$ cKOs. Our work suggests a model whereby NUMB and NUMBL regulate cell cycle withdrawal (Figure 9B) and whereby NUMB and NUMBL loss results in aberrant cell proliferation (Figure 9C). NUMB and NUMBL bind Rab7 and ERBB2 (Figure 9A) to tran- sit-activated ERBB2 from the EE to the LE en route to a degradative pathway, thus downregulating ERBB2 signaling and leading to cell cycle withdrawal. In the absence of NUMB and NUMBL, activated ERBB2 is retained within the EE, functioning as a signalosome (46) and resulting in increased activation/phosphorylation of STAT5. STAT5 directly interacts with YAP1 to result in YAP1 nuclear localization and activation of a YAP1-mediated proliferative program.

Four alternative splice isoforms of NUMB are expressed in a spatiotemporally distinct manner (10). We found that NUMB p65 and p71 are most likely to work at the EE/LE interface, as these 2 isoforms selectively interacted with Rab7 (Supplemental Figure 7A). We also found that NUMB p65 demonstrated strong affinity for NUMBL (Supplemental Figure 7A), suggesting that NUMB and NUMBL may work, in part, as heterodimers (Figure 9A).

In both $\mathrm{Nb} / \mathrm{NbL} \mathrm{cKO}$ trabeculae and $\mathrm{Nb} / \mathrm{NbL} \mathrm{KO} \mathrm{HeLa}$ cells, the number of LEs was decreased. Additionally, in Nb/NbL KO HeLa cells, we observed a significant increase in both the size and number of EEs ( $P=0.034$ and 0.005 , respectively). These observations suggest that a NUMB/NUMBL/Rab7 complex may be required for biogenesis of the LE/Lys, as Rab5 is required for EE biogenesis (47). Live cell imaging studies will be required in the future to fully understand how NUMB and NUMBL may affect vesicle biogenesis and trafficking.

Although both STAT5 and YAP1 mediate cell growth and oncogenic transformation, their interaction has not previously been described. Increased phosphorylation of STAT5 results in nuclear retention (48), and therefore interaction of PSTAT5 with YAP1 would be expected to result in nuclear retention of YAP1. Intriguingly, recent ChIP studies for YAP1 in a cardiomyocyte cell line indicated significant enrichment for STAT5-binding sites within the vicinity of YAP1-bound sites (49).

During formation of trabeculae, ERBB2 is required for initial delamination, directional migration, and proliferation (50). Factors regulating downregulation of ERBB2 in trabeculae are unknown. Here, we found selective enrichment of $\mathrm{Nb} / \mathrm{Nbl}$ in trabecular myocardium and also found that loss of $\mathrm{Nb} / \mathrm{Nbl}$ resulted in increased activation of ERBB2 and increased proliferation of trabeculae. Ectopically expressed or activated ERBB2 promotes proliferation of differentiated adult cardiomyocytes (32, 51, 52). Like ERBB2, activated YAP1 also induces proliferation of adult cardiomyocytes $(20,21)$. Our finding that YAP1 nuclear localization is downstream of ERBB2 activation suggests that this pathway may also override cell cycle withdrawal in adult cardiomyocytes.

Loss of either ERBB2 or YAP1 in cardiomyocytes results in arrested embryonic growth at E9.5 and embryonic lethality by E10.5, with mutants exhibiting cardiac hypoplasia (2, 43). ERBB2 activation in this context is mediated, at least in part, by ERK phosphorylation (53). In Nb/NbL cKOs, we observed increased pSTAT5, but no increased pERK. It remains to be seen whether increased pSTAT5 represents overactivation of a pathway normally activated downstream of ERBB2 during trabeculation or ectopic activation of a pathway not normally operative during trabeculation. Global knockout of both Sta$t 5 a$ and $S t a t 5 b$ does not result in embryonic lethality, but rather $30 \%$ lethality within 48 hours following birth of undetermined causes (54). Thus, loss of Stat $5 a$ and Stat $5 b$ does not mimic phenotypes of Erbb2 or Yap1 myocardial knockouts. Together, these 
observations suggest that STAT5 activation is not required for early heart development, although redundancy with other STAT factors, such as STAT1, cannot be ruled out, as STAT1 has been shown to interact with YAP1 $(55,56)$.

Recent studies have identified NUMB as a tumor suppressor $(11,12)$. Our data reveal insights as to how NUMB and NUMBL may act as tumor suppressors, promoting termination of growth factor signaling by interacting with Rab7 to facilitate targeting of ERBB2, and potentially other oncogenic growth factor receptors, to the LE for lysosomal degradation. Our work also uncovers unsuspected interactions between ERBB2 and YAP1, and STAT5 and YAP1. Aberrant activation of ERBB2 (57), aberrant phosphorylation of STAT5 (58), and aberrant nuclear localization of YAP1 (59) have been observed in multiple cancer settings and are targets of cancer therapy. Our observation that STAT5 activation can override Hippo inhibition of YAP1 may be of particular relevance to therapeutic targeting, suggesting it will be of future interest for examining dual STAT5/YAP1 activation.

\section{Methods}

Mice. All animals were maintained and experiments were performed in accordance with institutional guidelines at the University of California, San Diego. All mouse lines used in this study are listed in Supplemental Methods. $\mathrm{Nb} / \mathrm{Nbl}$ null mutants were generated by crossing $N b^{f / f l} N b^{f / f l}$ with Meox2-Cre mice. Numb cKOs were generated by crossing TnT-Cre $N b^{\Delta / W T}$ with $N b^{f / f l}$ mice, while $\mathrm{Nb} / \mathrm{NbL}$ cKOs were generated by crossing TnT-Cre $\mathrm{Nb}^{\Delta / W T} \mathrm{Nbl}^{\Delta / \Delta}$ with $N b^{f l f l l} \mathrm{Nbl}^{\Delta / \Delta}$ mice.

Plasmid construction. Each full-length cDNA was synthesized from total RNA extracted from mouse tissues, purchased, or provided as detailed in Supplemental Methods. The cDNA fragments of each deletion mutant were amplified by inverse PCR, followed by PCR-mediated recombination. Detailed nucleotide sequences are listed in Supplemental Methods.

Immunofluorescence. Mouse embryos and HeLa cells were fixed in $4 \%$ paraformaldehyde (PFA); then mouse embryos were embedded in Tissue-Tek OCT compound. Mouse frozen sections or HeLa cells were permeabilized with $0.1 \%$ Triton $\mathrm{X}-100$ and subsequently subjected to immunostaining. All antibodies used for immunohistochemistry are described in detail in Supplemental Methods. Stained sections/cells were mounted with Dako fluorescence mounting medium and visualized using an Olympus confocal microscope (FV1000), Deltavision OMX microscope, or Zeiss LSM8 Airyscan. EdU (Life Technologies) was intraperitoneally injected 2 hours before embryo dissection and detected with Click-iT EdU Alexa Fluor 647 Imaging Kit (Life Technologies).

Gene knockdown experiments and reverse transfection. Each siRNA was synthesized by Life Technologies and reverse transfected into HeLa cells using Lipofectamine RNAiMAX Reagent (Life Technologies) as previously described (60). Cells were plated at a density of 90,000 cells/well on 8-well chamber slides (Lab-Tek) after coating with Fibronectin (F1141, Sigma-Aldrich). Sequence information for siRNAs is provided in Supplemental Table 2. Cells were fixed with $4 \%$ PFA 48 hours after reverse transfection for immunostaining. For STAT5 inhibition, STAT5 inhibitor ( $N^{\prime}$-((4-Oxo-4H-chromen-3-yl) methylene)nicotinohydrazide; Millipore) was added to cells at a final concentration of $100 \mu \mathrm{M} 3$ hours before fixation.
Purification of GST-fused Rab proteins and NUMB interaction studies. GST-tagged Rab5a, Rab7a, Rab7b, and Rab11a were purified from BL21-competent $E$. coli cells as detailed in Supplemental Methods. Meanwhile, 293T cells were transiently transfected with the vector-expressing FLAG-tagged NUMB. Cell lysates were mixed with GDP- or GTP $\gamma$ S-bound Rab5a, Rab7a, Rab7b, or Rab11a recombinant proteins. After overnight incubation, each mixture was subjected to GST pull-down, separated by SDS-PAGE, and analyzed with anti-FLAG antibody.

Transfection, immunoprecipitation, and Western blotting. 293T or HeLa cells were transfected using Lipofectamine LTX with Plus Reagent (Life Technologies). Cell lysates were harvested 48 hours after transfection and subjected to immunoprecipitation with anti-FLAG M2 affinity gel (Sigma-Aldrich). After washing, immunocomplexes were resolved by SDSPAGE, transferred to PVDF membranes (Bio-Rad Laboratories), and reacted with HRP-conjugated antibodies. Signals were detected using Pierce ECL Western Blotting Substrate (Thermo Scientific).

Mouse tissue extraction and Western blotting. Embryonic heart ventricles were lysed with PBS with $1 \%$ Triton X-100. After centrifugation, supernatants were subjected to immunoprecipitation with anti-ERBB2 monoclonal antibody (3B5, Abcam), followed by Western blotting, or directly subjected to Western blotting without immunoprecipitation. Antibodies used are listed in Supplemental Methods. Western blots were quantitated with ImageJ software (NIH).

Quantitative analysis of intracellular vesicles. Z-stack series of pictures from 6 independent visual fields of 3 different samples were obtained for quantitation. To quantitate Lamp1-positive vesicles in trabecular myocytes, mouse tissue sections were quantitated with Image J software. For HeLa cell data, the number and/or mean volume of LAMP1or EEA1-positive endosomes and Pearson correlation coefficients were quantitated with Volocity 3D image analysis software. Parameters were set for the Volocity software to automatically detect each staining and were set equally for each sample to avoid any bias (Figure 5, B and E). Samples were prepared from 3 independent experiments.

Statistics. $P$ values were calculated using unpaired 2-tailed Student's $t$ test for single pairwise comparison, or 1-way or 2-way ANOVA with Tukey or Bonferroni-Holm post hoc analysis to correct for multiple comparisons. $P$ values of less than 0.05 were considered significant, and values shown are mean \pm SD.

Study approval. All studies were performed with the approval of the University of California San Diego Institutional Animal Care and Use Program (IACUC).

\section{Author contributions}

$\mathrm{MH}$ and SME were responsible for experimental design, data interpretation, and manuscript elaboration. $\mathrm{MH}$ and $\mathrm{YA}$ performed experiments. CJM, KFL, and JC contributed reagents and data interpretation.

\section{Acknowledgments}

We thank T. Nakamura (Kansai Medical University) and L. Selleri (Cornell University) for technical advice, G. Weinmaster (University of California, Los Angeles) for helpful discussions, C. Lai (Indiana University) and W. Zhong (Yale University) for providing antibodies, and T. Reya (University of California, San Diego) for providing TNR mice. This work was supported by grants to SME from the NIH, grants to MH from the Daiichi Sankyo Founda- 
tion of Life Science and the Uehara Memorial Foundation, and grants to KFL from the NIH (HD034534, NS060833, NS072031, AG010435, CA014195, AG042985 and AG047669), the Clayton Foundation, the Schlink Foundation, the Gemcon Family Foundation, and the Joe W. and Dorothy Dorsett Brown Foundation. Imaging was supported by UCSD Neuroscience Microscopy Shared Facility grant P30 NS047101.
Address correspondence to: Sylvia M. Evans, BRF2, Room 2A16, University of California, San Diego, 9500 Gilman Drive, La Jolla, California 92093, USA. Phone: 858.822.2452; E-mail: syevans@ucsd.edu.

MH's present address is: Department of Pharmacology, Kansai Medical School, Hirakata, Osaka, Japan.
1. Meyer D, Birchmeier C. Multiple essential functions of neuregulin in development. Nature. 1995;378(6555):386-390.

2. Lee KF, Simon H, Chen H, Bates B, Hung MC, Hauser C. Requirement for neuregulin receptor erbB2 in neural and cardiac development. Nature. 1995;378(6555):394-398.

3. Gassmann $\mathrm{M}$, et al. Aberrant neural and cardiac development in mice lacking the ErbB4 neuregulin receptor. Nature. 1995;378(6555):390-394.

4. Zhang W, Chen H, Qu X, Chang CP, Shou W. Molecular mechanism of ventricular trabeculation/compaction and the pathogenesis of the left ventricular noncompaction cardiomyopathy (LVNC). Am J Med Genet C Semin Med Genet. 2013;163C(3):144-156.

5. Pece S, Confalonieri S, R Romano P, Di Fiore PP. NUMB-ing down cancer by more than just a NOTCH. Biochim Biophys Acta. 2011;1815(1):26-43.

6. Bonifacino JS. Adaptor proteins involved in polarized sorting. J Cell Biol. 2014;204(1):7-17.

7. Kandachar V, Roegiers F. Endocytosis and control of Notch signaling. Curr Opin Cell Biol. 2012;24(4):534-540.

8. Couturier L, Trylinski M, Mazouni K, Darnet L, Schweisguth $\mathrm{F}$. A fluorescent tagging approach in Drosophila reveals late endosomal trafficking of Notch and Sanpodo. JCell Biol. 2014;207(3):351-363.

9. Caswell PT, Vadrevu S, Norman JC. Integrins: masters and slaves of endocytic transport. Nat Rev Mol Cell Biol. 2009;10(12):843-853.

10. Gulino A, Di Marcotullio L, Screpanti I. The multiple functions of Numb. Exp Cell Res. 2010;316(6):900-906.

11. Hong J, et al. The tumor suppressive role of NUMB isoform 1 in esophageal squamous cell carcinoma. Oncotarget. 2014;5(14):5602-5614.

12. Sima J, Zhang B, Yu Y, Sima X, Mao Y. Overexpression of Numb suppresses growth, migration, and invasion of human clear cell renal cell carcinoma cells. Tumour Biol. 2015;36(4):2885-2892.

13. Jiao K, et al. An essential role of Bmp4 in the atrioventricular septation of the mouse heart. Genes Dev. 2003;17(19):2362-2367.

14. Zilian O, et al. Multiple roles of mouse Numb in tuning developmental cell fates. Curr Biol. 2001;11(7):494-501.

15. Wilson A, et al. Normal hemopoiesis and lymphopoiesis in the combined absence of numb and numblike. JImmunol. 2007;178(11):6746-6751.

16. Muzumdar MD, Tasic B, Miyamichi K, Li L, Luo L. A global double-fluorescent Cre reporter mouse. Genesis. 2007;45(9):593-605.

17. Yang J, et al. Inhibition of Notch 2 by Numb/ Numblike controls myocardial compaction in the heart. Cardiovasc Res. 2012;96(2):276-285.

18. Zhao C, et al. Numb family proteins are essential for cardiac morphogenesis and progenitor differ- entiation. Development. 2014;141(2):281-295.

19. Hirai M, Chen J, Evans SM. Tissue-Specific Cell Cycle Indicator Reveals Unexpected Findings for Cardiac Myocyte Proliferation. Circ Res. 2016;118(1):20-28.

20. von Gise A, et al. YAP1, the nuclear target of Hippo signaling, stimulates heart growth through cardiomyocyte proliferation but not hypertrophy. Proc Natl Acad Sci USA. 2012;109(7):2394-2399.

21. Xin M, et al. Hippo pathway effector Yap promotes cardiac regeneration. Proc Natl Acad Sci USA. 2013;110(34):13839-13844.

22. Le Roux I, Konge J, Le Cam L, Flamant P, Tajbakhsh S. Numb is required to prevent p53-dependent senescence following skeletal muscle injury. Nat Commun. 2015;6:8528.

23. Wu B, et al. Inducible cardiomyocyte-specific gene disruption directed by the rat Tnnt 2 promoter in the mouse. Genesis. 2010;48(1):63-72.

24. Yu FX, Guan KL. The Hippo pathway: regulators and regulations. Genes Dev. 2013;27(4):355-371.

25. Zhao B, et al. Inactivation of YAP oncoprotein by the Hippo pathway is involved in cell contact inhibition and tissue growth control. Genes Dev. 2007;21(21):2747-2761.

26. Mizutani K, Yoon K, Dang L, Tokunaga A, Gaiano $\mathrm{N}$. Differential Notch signalling distinguishes neural stem cells from intermediate progenitors. Nature. 2007;449(7160):351-355.

27. Grego-Bessa J, et al. Notch signaling is essential for ventricular chamber development. Dev Cell. 2007;12(3):415-429.

28. Liu Z, et al. The intracellular domains of Notch1 and Notch2 are functionally equivalent during development and carcinogenesis. Development. 2015;142(14):2452-2463.

29. Ruff-Jamison S, Chen K, Cohen S. Epidermal growth factor induces the tyrosine phosphorylation and nuclear translocation of Stat 5 in mouse liver. Proc Natl Acad Sci USA.1995;92(10):4215-4218.

30. Olayioye MA, Beuvink I, Horsch K, Daly JM, Hynes NE. ErbB receptor-induced activation of stat transcription factors is mediated by Src tyrosine kinases. J Biol Chem. 1999;274(24):17209-17218.

31. Ward TM, et al. Truncated p110 ERBB2 induces mammary epithelial cell migration, invasion and orthotopic xenograft formation, and is associated with loss of phosphorylated STAT5. Oncogene. 2013;32(19):2463-2474.

32. Yutzey KE. Regenerative biology: Neuregulin 1 makes heart muscle. Nature. 2015;520(7548):445-446.

33. Goh LK, Sorkin A. Endocytosis of receptor tyrosine kinases. Cold Spring Harb Perspect Biol. 2013;5(5):a017459.

34. Chen JW, Murphy TL, Willingham MC, Pastan I, August JT. Identification of two lysosomal membrane glycoproteins. JCell Biol. 1985;101(1):85-95.
35. Gumbiner BM, Kim NG. The Hippo-YAP signaling pathway and contact inhibition of growth. JCell Sci. 2014;127(Pt 4):709-717.

36. Ran FA, Hsu PD, Wright J, Agarwala V, Scott DA, Zhang F. Genome engineering using the CRISPR-Cas9 system. Nat Protoc. 2013;8(11):2281-2308.

37. Mu FT, et al. EEA1, an early endosome-associated protein. EEA1 is a conserved alpha-helical peripheral membrane protein flanked by cysteine "fingers" and contains a calmodulin-binding IQ motif. J Biol Chem. 1995;270(22):13503-13511.

38. Ceresa BP. Regulation of EGFR endocytic trafficking by rab proteins. Histol Histopathol. 2006;21(9):987-993.

39. Stenmark H. Rab GTPases as coordinators of vesicle traffic. Nat Rev Mol Cell Biol. 2009;10(8):513-525.

40. Rink J, Ghigo E, Kalaidzidis Y, Zerial M. Rab conversion as a mechanism of progression from early to late endosomes. Cell. 2005;122(5):735-749.

41. Christoforidis S, Zerial M. Purification and identification of novel Rab effectors using affinity chromatography. Methods. 2000;20(4):403-410.

42. Iyer J, Reich NC. Constitutive nuclear import of latent and activated STAT5 a by its coiled coil domain. FASEB J. 2008;22(2):391-400.

43. Xin $\mathrm{M}$, et al. Regulation of insulin-like growth factor signaling by Yap governs cardiomyocyte proliferation and embryonic heart size. Sci Signal. 2011;4(196):ra70.

44. Zhang N, et al. The Merlin/NF2 tumor suppressor functions through the YAP oncoprotein to regulate tissue homeostasis in mammals. Dev Cell. 2010;19(1):27-38.

45. Crone SA, et al. ErbB2 is essential in the prevention of dilated cardiomyopathy. Nat Med. 2002;8(5):459-465.

46. Dobrowolski R, De Robertis EM. Endocytic control of growth factor signalling: multivesicular bodies as signalling organelles. Nat Rev Mol Cell Biol. 2011;13(1):53-60.

47. Zeigerer A, et al. Rab5 is necessary for the biogenesis of the endolysosomal system in vivo. Nature. 2012;485(7399):465-470.

48. Reich NC. STATs get their move on. JAKSTAT. 2013;2(4):e27080.

49. Lin Z, et al. Pi3kcb links Hippo-YAP and PI3K-AKT signaling pathways to promote cardiomyocyte proliferation and survival. Circ Res. 2015;116(1):35-45.

50. Liu J, et al. A dual role for ErbB2 signaling in cardiac trabeculation. Development. 2010;137(22):3867-3875.

51. D'Uva G, et al. ERBB2 triggers mammalian heart regeneration by promoting cardiomyocyte dedifferentiation and proliferation. Nat Cell Biol. 2015;17(5):627-638.

52. Gemberling M, Karra R, Dickson AL, Poss KD. $\mathrm{Nrg} 1$ is an injury-induced cardiomyocyte mitogen for the endogenous heart regeneration pro- 
gram in zebrafish. Elife. 2015;4.

53. Lai D, et al. Neuregulin 1 sustains the gene regulatory network in both trabecular and nontrabecular myocardium. Circ Res. 2010;107(6):715-727.

54. Teglund S, et al. Stat5a and Stat5b proteins have essential and nonessential, or redundant, roles in cytokine responses. Cell. 1998;93(5):841-850.

55. Walker SR, Xiang M, Frank DA. Distinct roles of STAT3 and STAT5 in the pathogenesis and targeted therapy of breast cancer. Mol Cell Endo- crinol. 2014;382(1):616-621.

56. Kristensen AR, Gsponer J, Foster LJ. A high-throughput approach for measuring temporal changes in the interactome. Nat Methods. 2012;9(9):907-909.

57. Ursini-Siegel J, Schade B, Cardiff RD, Muller WJ. Insights from transgenic mouse models of ERBB2-induced breast cancer. Nat Rev Cancer. 2007;7(5):389-397.

58. Furqan M, Akinleye A, Mukhi N, Mittal V, Chen
Y, Liu D. STAT inhibitors for cancer therapy. JHematol Oncol. 2013;6:90.

59. Zhao B, Li L, Lei Q, Guan KL. The Hippo-YAP pathway in organ size control and tumorigenesis: an updated version. Genes Dev. 2010;24(9):862-874.

60. Hirai M, Horiguchi M, Ohbayashi T, Kita T, Chien KR, Nakamura T. Latent TGF-beta-binding protein 2 binds to DANCE/fibulin-5 and regulates elastic fiber assembly. $E M B O J$. 2007;26(14):3283-3295. 